\title{
Mars, Phobos, and Deimos Sample Return Enabled by ARRM Alternative Trade Study Spacecraft
}

\author{
Jacob A. Englander*, Matthew A. Vavrina ${ }^{\dagger}$ Bo Naasz $\stackrel{\ddagger}{\ddagger}$ Raymond G. Merill, Min Qu ${ }^{\llbracket}$
}

The Asteroid Robotic Redirect Mission (ARRM) has been the topic of many mission design studies since 2011. ${ }^{1}$ The reference ARRM spacecraft uses a powerful solar electric propulsion (SEP) system and a bag device to capture a small asteroid from an Earth-like orbit and redirect it to a distant retrograde orbit (DRO) around the moon. The ARRM Option B spacecraft uses the same propulsion system and multi-Degree of Freedom (DoF) manipulators device to retrieve a very large sample (thousands of kilograms) from a 100+ meter diameter farther-away Near Earth Asteroid (NEA). This study will demonstrate that the ARRM Option B spacecraft design can also be used to return samples from Mars and its moons - either by acquiring a large rock from the surface of Phobos or Deimos, and/or by rendezvousing with a sample-return spacecraft launched from the surface of Mars.

\section{Introduction}

The Asteroid Robotic Redirect Mission (ARRM) Option A concept, first introduced in $2011,{ }^{1}$ is a mission design to capture and return a small near-Earth asteroid (NEA) to cislunar space. The ARRM Option B concept is a similar spacecraft, but designed to return a very large sample from a more difficult to reach NEA. ${ }^{2}$ In this work we show that the spacecraft designed for ARRM Option B is also well-suited to sample return from Mars and its moons. This work presents low-thrust interplanetary trajectories from cislunar space to Mars and back, including descent from the Martian sphere of influence (SOI) to the desired orbit altitude and ascent to the SOI after the sample retrieval is complete. Missions are presented to Phobos, Deimos, and low-Mars orbit (LMO), the latter from which the spacecraft could retrieve a sample capsule launched from the surface of Mars.

Boulder returns from Deimos or Phobos, or a Mars sample return are of notable value. The two moons are considered potential bases for manned teleoperations outposts, and serve as stepping stones to eventual human exploration of Mars (boots on ground). ${ }^{3,4}$ A precursor mission substantially minimizes the risk of any subsequent human mission to these bodies, enabling an examination of their composition. A robotic return of moon material would also help to answer questions as to their origin. Similarly, a Mars sample return has been charted as a critical step on the path to placing humans on the planet. It is the highest priority flagship mission identified in the 2013-2022 planetary science decadal survey. ${ }^{5}$ Asteroid robotic redirect vehicle (ARRV) trajectory performance is examined for viability of rendezvousing with a sample in a $400 \mathrm{~km}$ altitude orbit that is launched from the surface by another system.

Mission design is carried out using the Evolutionary Mission Trajectory Generator (EMTG), NASA Goddard Space Flight Center (GSFC)'s low-thrust trajectory optimization tool. ${ }^{6,7,8,9,10,11,12}$ This work presents the Mars System Sample Return (MSSR) problem as a global optimization problem in the EMTG framework and models the complex relationship between the mass of the sample, the mission time constraints, and the trajectory. EMTG is used to design the interplanetary phases of the trajectory and the Edelbaum

\footnotetext{
*Aerospace Engineer, Navigation and Mission Design Branch, NASA Goddard Space Flight Center, Greenbelt, MD, 20771, USA, Member AIAA

†Senior Systems Engineer, a.i. solutions, Inc., 10001 Derekwood Ln. Suite 215, Lanham, MD, 20706, USA

¥System Engineering Manager, Satellite Servicing Capabilities Office (SSCO), NASA GSFC, Mail Stop 408, Greenbelt, MD 20771, AIAA Member.

$\S$ Aerospace Engineer, Space Mission Analysis Branch, NASA Langley Research Center, Hampton, VA, 23681, USA, Senior Member AIAA

『Staff Scientist, Analytical Mechanics Associates, Inc., 21 Enterprise Parkway, Suite 300, Hampton, VA 23666-6413
} 
approximation is used to model the flight time and propellant use from the SOI to Deimos/Phobos/LMO and back to the SOI.

\section{Mission Modeling}

\section{II.A. Mission Architecture}

The MSSR architecture considered this work is based heavily on the ARRM Option B mission concept. ARRM Option B consists of a large solar-electric propulsion (SEP) flight module with an attached mission module. The combined vehicle is referred to as the asteroid robotic redirect vehicle (ARRV). The mission module is made up of a set of gripper arms, a mechanism to anchor to the target, a structure on which to mount them, and avionics. In ARRM Option B, the grippers are used to acquire a boulder from the surface of a large near-Earth object (NEO) and hold that boulder all the way back to Earth. The MSSR paradigm is identical except that the grippers are used either to acquire a boulder from the surface of Phobos or Deimos, or to grapple a vehicle launched from the surface of Mars.

The MSSR spacecraft is powered by a pair of large solar arrays that supplies $51 \mathrm{~kW}$ of power at beginning of life (BOL) at $1 \mathrm{AU}$. The performance of the arrays as a function of distance from the sun is modeled using EMTG's power model ${ }^{10}$ with a set of coefficients for triple-junction gallium arsenide (TJGA) cells and the array is assumed to lose $1 \%$ of its performance for each year of flight. The spacecraft bus consumes a constant $0.5 \mathrm{~kW}$ and a $15 \%$ margin is applied on input power to the propulsion system.

There are two versions of the MSSR propulsion system, both based on a high-power Hall thruster. The first version of the propulsion system is modeled as a single "super-thruster" running at $2000 \mathrm{~s}$ specific impulse $\left(I_{s p}\right)$ and $55 \%$ system efficiency that accepts up to $40 \mathrm{~kW}$ of input power. Thrust is computed using EMTG's propulsion mode ${ }^{10}$ as a function of available power. The second version is also modeled as a "super-thruster" but runs at $3000 \mathrm{~s} I_{s p}$ with $60 \%$ system efficiency. The $2000 \mathrm{~s}$ version is slightly heavier than the $3000 \mathrm{~s}$ version and adds $150 \mathrm{~kg}$ to the spacecraft. Both propulsion systems are composed of the same hardware, but the $2000 \mathrm{~s}$ system is run at $400 \mathrm{~V}$ while the $3000 \mathrm{~s}$ system is run at $800 \mathrm{~V}$. There are two additional thrusters in the $2000 \mathrm{~s}$ system. Both propulsion systems are run at a $90 \%$ duty cycle. An $11 \%$ margin is carried on the xenon propellant.

There are two launch paradigms for MSSR. The first is to launch the spacecraft on the Space Launch System (SLS) Block 1a launch vehicle to a C3 of up to $27 \mathrm{~km}^{2} / \mathrm{s}^{2}$. The SLS can deliver up to $16072 \mathrm{~kg}$ to this energy based on current notional performance curve. A 30 day coast period is enforced after launch for spacecraft systems checkout. The alternative launch paradigm is to launch to trans-lunar injection (TLI) on a Falcon Heavy $(\mathrm{FH})$ and then perform a double lunar flyby sequence. FH is assumed to be capable of launching $13200 \mathrm{~kg}$ to a $\mathrm{C} 3$ of $-2.0 \mathrm{~km}^{2} / \mathrm{s}^{2}$ which enables the lunar flyby sequence. The double flyby delivers the spacecraft to a C3 of $2.0 \mathrm{~km}^{2} / \mathrm{s}^{2}$ and takes 60 days, which conveniently is enough time to perform systems checkout so no coast period is required after the spacecraft escapes from cislunar space at the second flyby. ${ }^{13}$ While the Earth departure portion of the trajectory is critical, it is not the focus of this study and the results shown allow for a departure C3 values that are less than or equal to $2.0 \mathrm{~km}^{2} / \mathrm{s}^{2}$. The practicality of this assumption has been verified by reproducing the trajectories generated in EMTG in end-to-end simulations in the high-fidelity modeling of the Copernicus software. ${ }^{14}$

Upon arrival at Mars, the spacecraft descends to the altitude of Phobos, Deimos, or a $400 \mathrm{~km}$ altitude LMO depending on the desired mission, spends between 200 and 600 days, and then ascends to the boundary of the Mars SOI and then returns to Earth. A constraint is applied to Earth return such that the incoming C3 is less than or equal to $2.0 \mathrm{~km}^{2} / \mathrm{s}^{2}$, which allows for a lunar flyby and then entry into a distant retrograde orbit (DRO). The $\Delta v$ necessary to insert into the DRO from the arrival trajectory is approximated at 100 $\mathrm{m} / \mathrm{s}$. Finally, the dry mass of the spacecraft is constrained to be no less than $5170 \mathrm{~kg}$ for the $2000 \mathrm{~s} I_{s p}$ version and no less than $5070 \mathrm{~kg}$ for the $3000 \mathrm{~s} I_{s p}$ version. These parameters are listed concisely in Table 1. No other information was supplied to EMTG.

\section{II.B. The Sims-Flanagan Transcription}

The Sims-Flanagan transcription is a widely used method in which the continuous-thrust trajectory is discretized into many small time steps, and the thrust applied during each time step is approximated as a small impulse placed at the center of the time step. The trajectory is propagated between control points by solving Kepler's problem. ${ }^{15}$ The Sims-Flanagan transcription, when used with an nonlinear programming (NLP) 


\begin{tabular}{|c|c|}
\hline \multicolumn{2}{|l|}{ Power Assumptions } \\
\hline BOL Power at $1 \mathrm{AU}$ & $51 \mathrm{~kW}$ \\
\hline Array performance model & TJGA \\
\hline Array decay rate & $1 \%$ per year \\
\hline Spacecraft bus power & $0.5 \mathrm{~kW}$ \\
\hline Power Margin & $15 \%$ \\
\hline \multicolumn{2}{|l|}{ Propulsion Assumptions } \\
\hline Thruster model & Fixed $I_{s p}$ and system efficiency \\
\hline Input power bounds & $0-40 \mathrm{~kW}$ \\
\hline Thruster performance & $\begin{array}{l}2000 \mathrm{~s} I_{s p} \text { with } 55 \% \text { efficiency } \\
3000 \mathrm{~s} I_{s p} \text { with } 60 \% \text { efficiency }\end{array}$ \\
\hline Duty cycle & $90 \%$ \\
\hline Propellant margin & $11 \%$ \\
\hline \multicolumn{2}{|l|}{ Launch Assumptions } \\
\hline Maximum departure hyperbolic excess energy (C3) & $\begin{array}{l}2 \mathrm{~km}^{2} / \mathrm{s}^{2}(\mathrm{FH}) \\
27 \mathrm{~km}^{2} / \mathrm{s}^{2}(\mathrm{SLS})\end{array}$ \\
\hline Maximum launch mass & $\begin{array}{l}13153 \mathrm{~kg}\left(\mathrm{FH} \text { with } 2000 \mathrm{~s} I_{s p}\right) \\
13169 \mathrm{~kg}\left(\mathrm{FH} \text { with } 3000 \mathrm{~s} I_{s p}\right) \\
16072 \mathrm{~kg}(\mathrm{SLS})\end{array}$ \\
\hline Declination of Launch Asymptote (DLA) bounds & $+/-28.5^{\circ}$ \\
\hline Post-launch forced coast & $\begin{array}{l}30 \text { days }(\mathrm{SLS}) \\
\text { none }(\mathrm{FH})\end{array}$ \\
\hline \multicolumn{2}{|l|}{ Mars stay assumptions } \\
\hline Desired altitude & $\begin{array}{l}20063 \mathrm{~km} \text { (Phobos) } \\
5844 \mathrm{~km} \text { (Deimos) } \\
400 \text { (LMO) }\end{array}$ \\
\hline $\begin{array}{l}\text { Stay time at desired altitude } \\
\text { Earth Return Assumptions }\end{array}$ & $200-600$ days \\
\hline Maximum arrival C3 & $2.0 \mathrm{~km}^{2} / \mathrm{s}^{2}$ \\
\hline Post-mission $\Delta v$ & $100 \mathrm{~m} / \mathrm{s}$ \\
\hline Minimum spacecraft final mass & $\begin{array}{l}5170 \mathrm{~kg}\left(2000 \mathrm{~s} I_{s p} \text { case }\right) \\
5020 \mathrm{~kg}\left(3000 \mathrm{~s} I_{s p} \text { case }\right)\end{array}$ \\
\hline
\end{tabular}

Table 1: ARRM Option B Assumptions 
solver such as Sparse Nonlinear Optimizer (SNOPT) and a suitable initial guess, is very fast and robust. It is considered to be a "medium-fidelity" transcription and is used in existing software packages such as Mission Analysis Low-Thrust Optimization (MALTO), ${ }^{16}$ Gravity Assisted Low-thrust Local Optimization Program (GALLOP), ${ }^{17}$ and Parallel Global Multiobjective Optimizer (PaGMO). ${ }^{18}$

In the classical Sims-Flanagan transcription, the optimizer chooses the three components of an impulsive $\Delta \mathbf{v}$ vector at the center of each time-step. In order to improve the robustness of the solver, a modified transcription known as "up-to-unit vector control" is used in EMTG, where instead of choosing the $\Delta \mathbf{v}$ vector directly the optimizer instead chooses a control 3 -vector in $[-1.0,1.0]$ that is multiplied by the maximum $\Delta v$ that the spacecraft can produce in that time-step. The magnitude of the control vector is then bounded in the range $[0.0,1.0]$, i.e.,

$$
\Delta \mathbf{v}_{i}=\mathbf{u}_{i} \Delta v_{\max , i},\left\|\mathbf{u}_{i}\right\| \leq 1.0
$$

where

$$
\Delta v_{\max , i}=\frac{D n_{\text {available }} T_{\max }\left(t_{f}-t_{0}\right)}{m N}
$$

where $D$ is the thruster duty cycle, $n_{\text {available }}$ is the number of available thrusters, $T_{\max }$ is the maximum available thrust from one thruster, $t_{0}$ and $t_{f}$ are the beginning and ending times of the time step, $m$ is the mass of the spacecraft at the center of the time step, and $N$ is the number of time steps in the phase. This modified Sims-Flanagan transcription is used in MALTO, Parallel Global Multiobjective Optimizer (PAGMO), and EMTG.

In each phase of the mission, the trajectory is propagated forward from the first endpoint (i.e. planet) and backward from the second endpoint. The trajectory is propagated by solving Kepler's equation and the spacecraft mass is propagated by assuming a constant mass flow rate across the each time-step. The specific Kepler propagator algorithm used in EMTG is a Laguerre-Conway method. ${ }^{19,20}$ A set of nonlinear constraints are applied to ensure continuity in the center of the phase,

$$
\mathbf{s}_{m f}-\mathbf{s}_{m b}=\left[\begin{array}{lllllll}
\Delta x & \Delta y & \Delta z & \Delta v_{x} & \Delta v_{y} & \Delta v_{z} & \Delta m
\end{array}\right]=\mathbf{0}
$$

The optimizer also chooses the initial and final velocity vectors for each phase. If a phase begins with a launch, the magnitude of the initial velocity vector is used with a launch vehicle model to determine the initial mass of the spacecraft as described later in this work. If a phase begins with a planetary flyby, two nonlinear constraints are applied to ensure that the flyby is feasible. First, the incoming and outgoing velocity vectors with respect to the planet must be equal,

$$
v_{\infty}^{+}-v_{\infty}^{-}=0
$$

where $v_{\infty}^{-}$and $v_{\infty}^{+}$are the velocities before and after the flyby, respectively. Second, the spacecraft may not fly closer to the planet than some user-specified minimum flyby distance:

$$
\frac{\mu_{\text {planet }}}{v_{\infty}^{2}}\left[\frac{1}{\sin \left(\frac{\delta}{2}\right)}-1\right]-\left(r_{\text {planet }}+h_{\text {safe }}\right) \geq 0
$$

where

$$
\delta=\arccos \left[\frac{\mathbf{v}_{\infty}^{-} \cdot \mathbf{v}_{\infty}^{+}}{\left(v_{\infty}^{-}\right)^{2}\left(v_{\infty}^{+}\right)^{2}}\right]
$$

Here $\mu_{\text {planet }}$ is the gravitational parameter of the planet, $r_{\text {planet }}$ is the radius of the planet, $\delta$ is the flyby turn angle, and $h_{\text {safe }}$ is the user-defined minimum altitude.

Figure 1 is a diagram of a simple low-thrust mission to Jupiter with one Earth flyby using the multiple gravity assist with low-thrust (MGALT) model. The continuity constraints are deliberately left unsatisfied in the diagram to illustrate where they must be applied.

There are four significant advantages to using the Sims-Flanagan transcription. First, the optimal objective function value for a Sims-Flanagan trajectory design is usually very close to the optimal cost value for a higher-fidelity version of the same trajectory. Second, a low-thrust trajectory generated using the Sims-Flanagan transcription makes a very good initial guess for a higher-fidelity trajectory design. Third, 


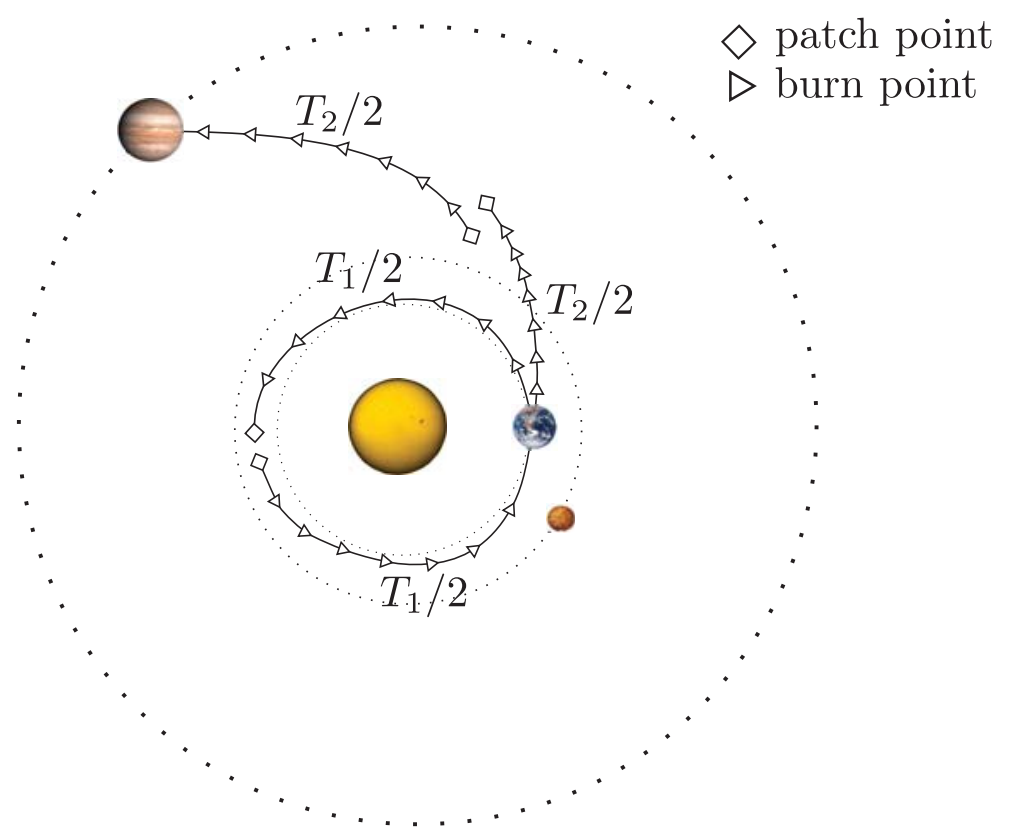

Figure 1: An Example Trajectory Using the Sims-Flanagan Transcription

the Sims-Flanagan transcription is very fast because it does not require numerical integration of differential equations. Fourth, the convergence of an NLP solver solving a Sims-Flanagan problem is very robust to poor initial guesses, making it ideal for an automated design approach. It is therefore very reasonable to use the Sims-Flanagan transcription for preliminary design of missions like MSSR.

\section{II.C. Spiral Low-Thrust Trajectory Modeling via Edelbaum's Method}

Low thrust orbit raising and lowering about a planetary body is often referred to as "spiraling" because the time-scale of the orbit is short relative to the length of the maneuver and so the spacecraft performs many revolutions about the planet before arriving at its desired altitude. When the trajectory is viewed it looks like a spiral. Because of the large number of revolutions it is impractical to design such a trajectory using the Sims-Flanagan transcription because far too many time-steps would be required and therefore the problem would become intractible. Fortunately there is a convenient approximation that makes the low-thrust orbit raising and lowering problem much easier.

Edelbaum ${ }^{21}$ showed that when the magnitude of the applied thrust is very low relative to gravity then the spacecraft's orbit may be approximated as being circular at all times. For "up spiral" trajectories, thrust is applied along the orbit velocity direction until the orbit reaches the boundary of the planets SOI where the orbit becomes hyperbolic with an excess velocity of 0 . Thrust is applied opposite the orbit velocity direction for "down spiral" trajectories. No plane change is necessary during either type of spiral trajectories because the excess velocity of 0 can be reached from any orbit plane of choice. The $\Delta v$ required to transfer from a higher orbit to a lower orbit is therefore simply the difference between the circular orbit speeds at the starting and ending altitudes, i.e.,

$$
\Delta v_{\text {Edelbaum }}=\sqrt{\frac{\mu_{\text {planet }}}{r_{0}}}-\sqrt{\frac{\mu_{\text {planet }}}{r_{f}}}
$$

where $r_{0}$ and $r_{f}$ are the radii of the starting and ending orbit, respectively. In EMTG a spiral arrival is determined by solving Equation 7 where $r_{0}$ is equal to the radius of the planet's SOI and $r_{f}$ is the radius of the desired final circular orbit. Conversely, for departure spirals $r_{0}$ is the radius of the initial circular orbit and $r_{f}$ is the radius of the planet's SOI.

The propellant necessary to accomplish the spiral may then be determined by,

$$
m_{\text {propellant }}=m_{\text {before-spiral }}\left(1-\exp \left(-1000 \frac{\Delta v_{\text {Edelbaum }}}{I_{\text {sp }} g_{0}}\right)\right)
$$


where $m_{\text {before-spiral }}$ is the mass of the spacecraft before the spiral orbit transfer and $g_{0}$ is the acceleration due to gravity at sea level on Earth, $9.80665 \mathrm{~m} / \mathrm{s}^{2}$. The time required for the spiral may then be computed by determining how much time is required for the thruster to expel the necessary propellant,

$$
t_{\text {Edelbaum }}=\frac{m_{\text {propellant }}}{\dot{m}}
$$

where $\dot{m}$ is the mass flow rate of the thruster, which is a function of the available electric power according to EMTG's propulsion and power modeling system. ${ }^{10}$ In EMTG, the available power and therefore the thrust, $I_{s p}$, and mass flow rate are held constant across the spiral orbit transfer, fixed to the power available at the beginning of the maneuver. Therefore for an arrival spiral, EMTG computes the power at the epoch where the spacecraft is at the edge of the planet's SOI and for a departure spiral EMTG computes the power available at the epoch where the spacecraft is in its initial circular parking orbit about the planet.

The Edelbaum approximation, while not an exact model of the spiral descent and ascent problem, is a very good approximation suitable for preliminary design. In this work the Edelbaum equations are used to model the motion of the spacecraft from the radius of Mars's SOI to the orbital altitudes of Phobos and Deimos, and to LMO, and back up to the SOI boundary.

\section{Trajectory Optimization}

\section{III.A. The Rendezvous Sample-Return Problem with Fixed Pickup Mass}

The first case considered in this work is zeroth-order approximation of the true Mars sample return problem. The spacecraft travels to Mars in the heliocentric frame, disappears, and re-emerges some time later with its mass incremented by the difference between the size of sample collected and the mass of the propellant necessary to perform all maneuvers in the Mars reference frame, i.e.,

$$
m_{\text {Mars-departure }}=m_{\text {Mars-arrival }}+m_{\text {sample }}-m_{\text {propellant-used-at-Mars }}
$$

where $m_{\text {propellant-used-at-Mars }}$ is the mass required to descend to and return from the orbit of interest, i.e. that of Deimos, Phobos, or LMO. This formulation of the problem is most appropriate if $m_{\text {sample }}$ is varied parametrically and $m_{\text {propellant-used-at-Mars }}$ is calculated external to the trajectory program. The MSSR problem then becomes a straightforward two-journey out-and-back mission to Mars which can be designed in any of several existing low-thrust trajectory design programs. ${ }^{10,16,17,18}$

The Jacobian sparsity pattern for the rendezvous, fixed pickup mass MSSR problem with no planetary flybys is shown in Figure 2. The diagonal blue lines represent the dependence of the throttle constraint in each time-step on the control variables in that time-step. The horizontal solid blue bars represent the dependence of the phase match-point constraints on all of the control variables in each phase and on time. The sparse set of dots at the bottom represent the dependence of the return C3, return date, total flight time, and dry mass constraints on the launch date, the initial mass of the spacecraft, the flight times for each phase, and the stay time at Mars. The solitary blue dot in the center of the bottom row represents the dependence of the objective function, i.e. maximum return mass, on the mass at the beginning of the return journey. The pattern in Figure 2 does not take into account any dependencies introduced by the spiral descent and ascent because they are computed outside of the main trajectory optimization. Note that, if the off-line computation of the Edelbaum equations is omitted, this formulation also works for retrieving a sample from an asteroid as in the original ARRM Option B concept. This sparsity pattern is useful for comparison with those of other formulations of the MSSR problem later in this work. While the rendezvous, fixed pickup mass MSSR problem is convenient to formulate in most existing tools, it comes with a significant drawback. A series of nested parametric runs must be performed to explore the trade-space. First, for a variety of launch dates, outbound trajectories must be designed from Earth to Mars. An offline batch computation of the Edelbaum equations must then be conducted to find the time and propellant mass necessary to descend from the boundary of Mars's SOI to the target orbit. Pickup mass is then varied parametrically and, for each pickup mass, spacecraft arrival scenario at Mars, and desired stay time at Mars, a return trajectory must be computed. Let $N_{L D}$ be the number of launch dates in the parametric sweep, and let $N_{t \text {-outbound }}$ be the number of outbound flight time discretizations, $N_{P M}$ be the number of pickup mass discretizations, $N_{\text {stay }}$ be the number of stay time discretizations, and $N_{\text {return-date }}$ be the number of return date discretizations. Then, since the time and propellant mass required for the spiral descent and ascent are a function of the 


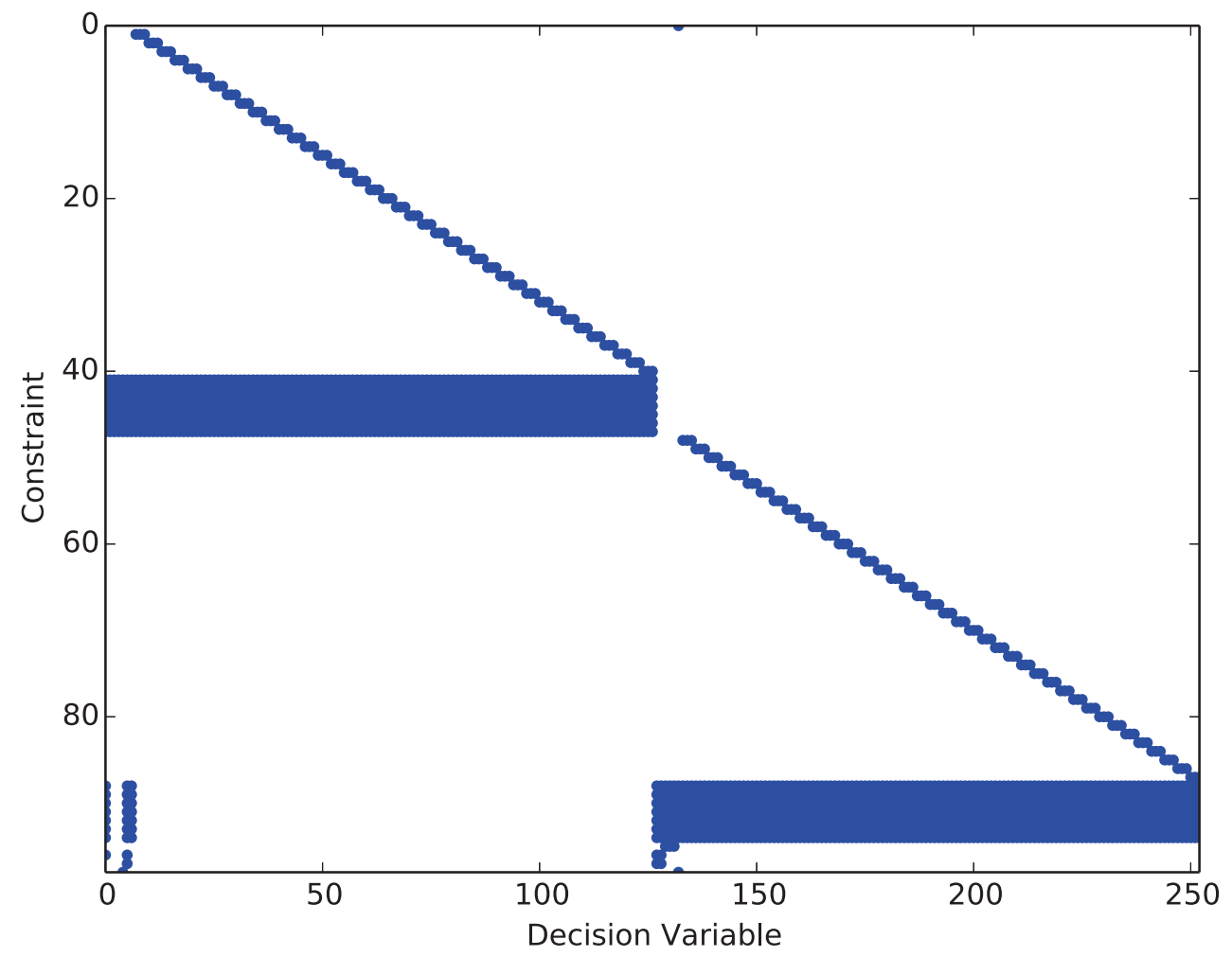

Figure 2: Sparsity Pattern for the Rendezvous Sample-Return Problem with Fixed Pickup Mass

other variables, the number of trajectories to be computed in the parametric sweep is:

$$
N_{\text {total }}=N_{L D} N_{\text {t-outbound }} N_{P M} N_{\text {stay }} N_{\text {return-date }}
$$

The expression in Equation 11 can quickly grow to an impractically large number of trajectories. It is therefore desirable to find a more efficient method to perform the search.

\section{III.B. The Sample-Return Problem with Fixed Pickup Mass and Spiral Modeling at Mars}

A better, but still not fully modeled, version of the MSSR problem can be constructed by including the computation of the Edelbaum (or other low-thrust spiral approximation) equations in the trajectory tool itself as is done in EMTG. ${ }^{10}$ This approach removes the necessity to perform any off-line computations and therefore allows the outbound and return components of the mission to be optimized in a single run, cutting down on the total number of runs of the trajectory optimization tool. However, in this formulation pickup mass is still varied parametrically and so the number of runs is still large:

$$
N_{\text {total }}=N_{L D} N_{P M} N_{\text {return-date }}
$$

The Jacobian sparsity pattern for the fixed pickup mass with spiral modeling formulation of the MSSR problem with no planetary flybys is shown in Figure 3. The entries which are common between the fixed mass, rendezvous problem and the fixed mass, spiral problem are shown in blue. The new entries which are introduced by including the spiral descent and ascent in the trajectory optimization are shown in red. The single red dot in the bottom left represents the new dependency of the flight time constraint on the launch date. This dependency occurs because the launch date influences the arrival date at Mars, which in turn influences the distance between Mars and the Sun at arrival, which in turn influences the available power for the spiral descent to the desired orbit and therefore the available thrust and $I_{s p}$. This dependency on solar distance influences the amount of time necessary for the spiral descent and ascent and therefore the total mission time. Similarly the line of three red dots next to the solitary red dot represent the influence of the outbound journey flight time on the Earth return date, the total flight time, and the spacecraft dry 
mass constraint. Like the launch date, the outbound flight time influences the date of arrival at Mars and therefore the available power and thus the flight time and propellant mass necessary to perform the spiral descent and ascent. These variables in turn influence the return date and total flight time as expected but also the total amount of propellant consumed that affects the dry mass constraint. These new dependencies were implicit in the fixed mass, rendezvous version of the MSSR problem but did not need to be considered by the optimizer. Switching to the fixed mass, spiral modeling version of the problem allows the total number of optimizer runs to be reduced but require that the optimizer properly process the relationship between time and mass in the spiral descent and ascent modeling.

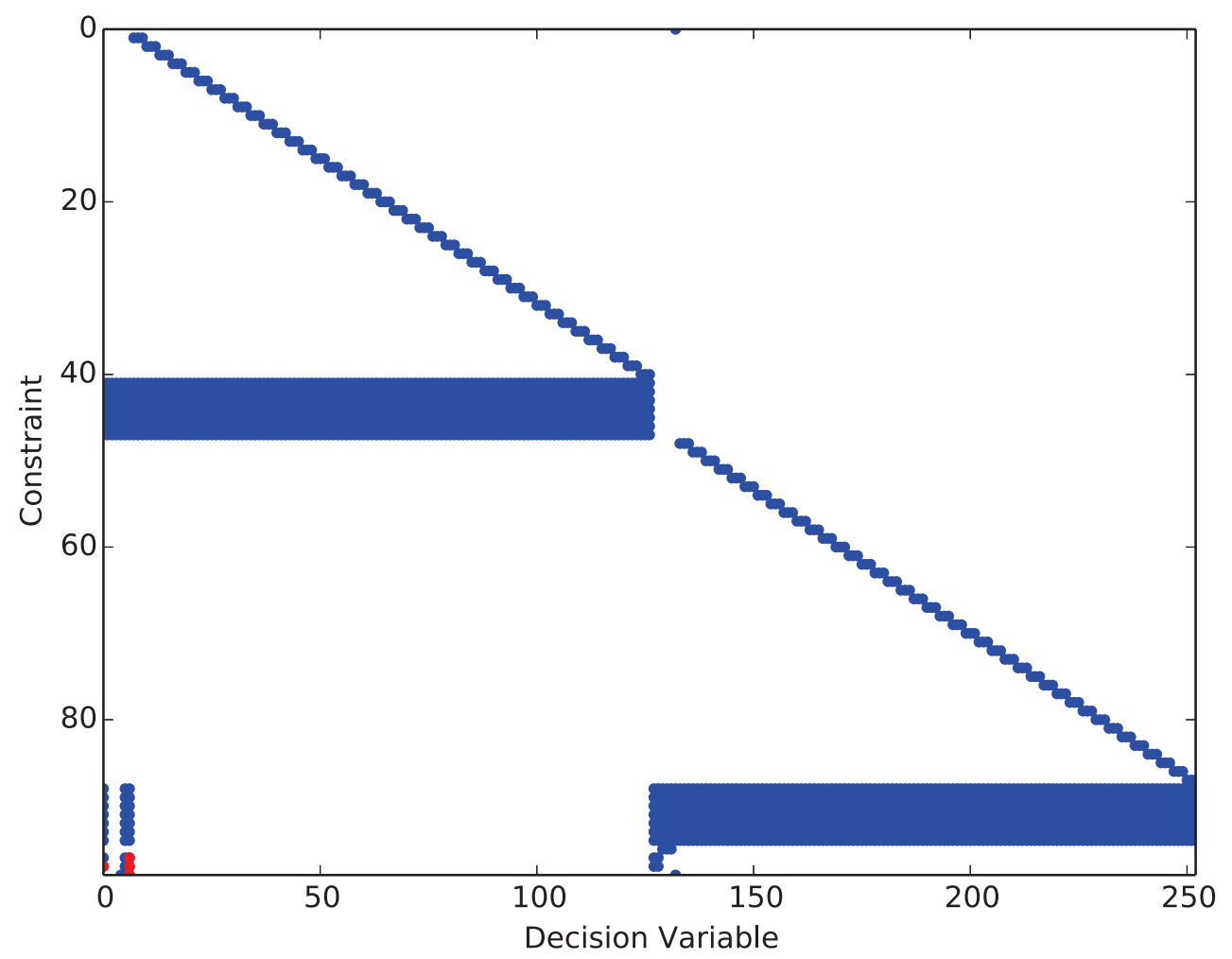

Figure 3: Sparsity Pattern for the Sample-Return Problem with Fixed Pickup Mass and Spiral Modeling

\section{III.C. The Sample-Return Problem with Variable Pickup Mass and Spiral Modeling at Mars}

While the fixed mass, spiral modeling version of the MSSR problem is simpler to use and can explore the trade space in fewer runs of the trajectory optimization program, it is still not ideal because the pickup mass must still be discretized. It is desirable to find a formulation of the MSSR problem that does not require any discretization at all except, if desired, on launch date and return date. This approach is implemented by allowing the optimizer to directly vary the pickup mass as is done in EMTG. ${ }^{10}$ There is therefore no need for a parametric sweep on mass and the number of runs is reduced further, to:

$$
N_{\text {total }}=N_{L D} N_{\text {return-date }}
$$

The Jacobian sparsity pattern for the MSSR problem with variable pickup mass, spiral descent/ascent modeling, and no planetary flybys is shown in Figure 4. The entries common to all three versions of the problem are shown in blue, and the entries common to the fixed-mass and variable-mass problems with spiral descent/ascent modeling are shown in purple. The new entries unique to the variable mass problem are shown in red. There are ten new entries. The first seven are a vertical line in the thick blue bar representing the dependencies of the return-journey match point constraints. These represent the dependency of the match point constraints on the variable pickup mass. The final three entries are immediately below the thick blue bar and represent the dependence of the Earth return date and total flight time on the pickup mass. These dependencies are introduced because when the pickup mass changes, the time required for the ascent spiral 
changes and influences the return date and total flight time. In addition, the pickup mass influences the amount of propellant necessary for the ascent spiral and therefore the dry mass constraint.

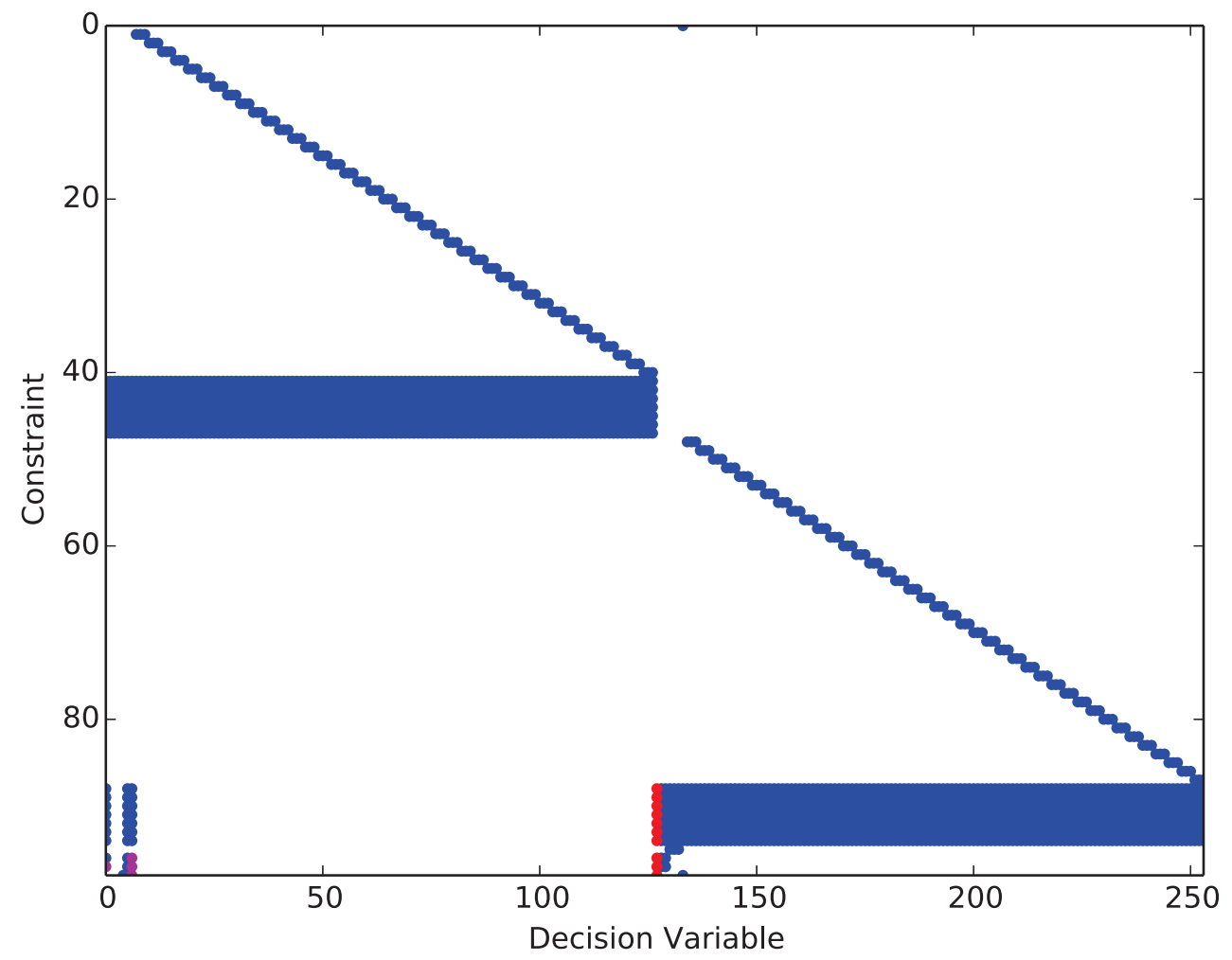

Figure 4: Sparsity Pattern for the Spiral Sample-Return Problem with Variable Pickup Mass

The variable pickup mass with spiral modeling formulation of the MSSR problem is the most complex of the three presented here and requires a tool that can properly model the additional dependencies but significantly reduces the number of parametric runs required. This is the most efficient, in terms of the human overhead required to solve the problem, approach to designing MSSR trajectories and is therefore implemented in EMTG.

\section{III.D. Stochastic Global Search via Monotonic Basin Hopping and SNOPT}

Like most other optimization problems, low-thrust trajectory design problems require an initial guess. Such initial guesses are often generated using Lambert's method, by solving for the optimal impulsive trajectory,by using shape-based trajectory approximations, or by experience-driven intuition. Once an initial guess is found for one version of the problem, the solution to the first problem may be used as an initial guess for related problems. For example if one finds a solution to the fixed-mass rendezvous MSSR problem with $2000 \mathrm{~kg}$ of return mass, that solution may be used as an initial guess for the same problem with $3000 \mathrm{~kg}$ of return mass. However there are drawbacks in this approach. First, the optimal low-thrust solution may not be in the neighborhood of the optimal solution to the low-fidelity Lambert version of the same problem. Second, sometimes the solution space bifurcates - that is, sometimes as the pickup mass increases multiple families of solutions can be found and if one uses a previous solution with lower pickup mass as an initial guess, one falls into an inferior solution family. Similarly, if one is solving the variable pickup mass problem, the optimizer will only converge to the locally optimal solution that represents the best in the solution family that was used as the initial guess.

Recent research in low-thrust trajectory optimization has led to the creation of stochastic search methods that do not require an initial guess. ${ }^{22,23,24,25,26,6,7,8,9,10,11,12}$ Such methods are ideal for the MSSR problem because they can be used to directly solve the low-thrust problem without the risk of introducing a bias by using a previous solution to a related problem or a low-fidelity approximation to the same problem. In addition, because each problem instance is now independent of all of the others, stochastic search methods 
allow many different versions of the MSSR problem to be solved simultaneously in parallel. The stochastic search method used in this work is monotonic basin hopping (MBH).

$\mathrm{MBH}^{27}$ is an algorithm for finding globally optimal solutions to problems with many local optima. MBH works on the principle that many real-world problems have a structure where individual local optima, or "basins" tend to cluster together into "funnels" where one local optimum is better than the rest. A problem may have several such funnels. MBH was originally developed to solve molecular conformation problems in computational chemistry, but has been demonstrated to be effective on various types of interplanetary trajectory problems. ${ }^{26,28,29,7,8,9}$

First, an initial point $\mathbf{x}$ is randomly chosen. The NLP solver is run using $\mathbf{x}$ as the initial guess. If the NLP solver finds a feasible solution, then that new point $\mathbf{x}^{*}$ is adopted as the new current point. If the NLP solver does not find a feasible solution, then a new random point is chosen. Once a feasible solution is found, MBH will attempt to "hop" from the feasible and locally optimal $\mathbf{x}^{*}$ to a better point. This is a two-step process: first a small random perturbation vector is added to $\mathbf{x}^{*}$, producing a new $\mathbf{x}^{\prime}$, and then the NLP solver is run. If the resulting solution is both feasible and superior to $\mathbf{x}^{*}$, then it is adopted as the new $\mathbf{x}^{*}$ and the hopping process begins again. Otherwise, MBH attempts a new hop from the current $\mathbf{x}^{*}$. Each feasible solution is stored in an archive.

$\mathrm{MBH}$ is run until either a specified number of iterations (trial points attempted) or a maximum CPU time is reached, at which point the best solution stored in the archive is returned as the solution to the outer-loop. The version of MBH used in this work has two parameters - the stopping criterion and the type of random step used to generate the perturbed points $\mathbf{x}^{\prime}$. In this work the random step is drawn from a bi-directional Pareto distribution with the Pareto parameter, $\alpha$, set to 1.5. The bi-directional Pareto distribution will usually generate small steps that allow MBH to exploit the local funnel around the current best solution. However some of the steps generated by the bi-directional Pareto distribution will be much larger, in some cases spanning the entire solution space. These larger steps allow MBH to explore the full problem. This approach is known to be robust on complex low-thrust problems. ${ }^{12}$

In addition, SNOPT occasionally freezes in the middle of a local optimization. This behavior disrupts the MBH global search, so EMTG contains a timer that ends any SNOPT run that continues for longer than some threshold time typically set to a few minutes. The pseudocode for MBH is listed in Algorithm 1.

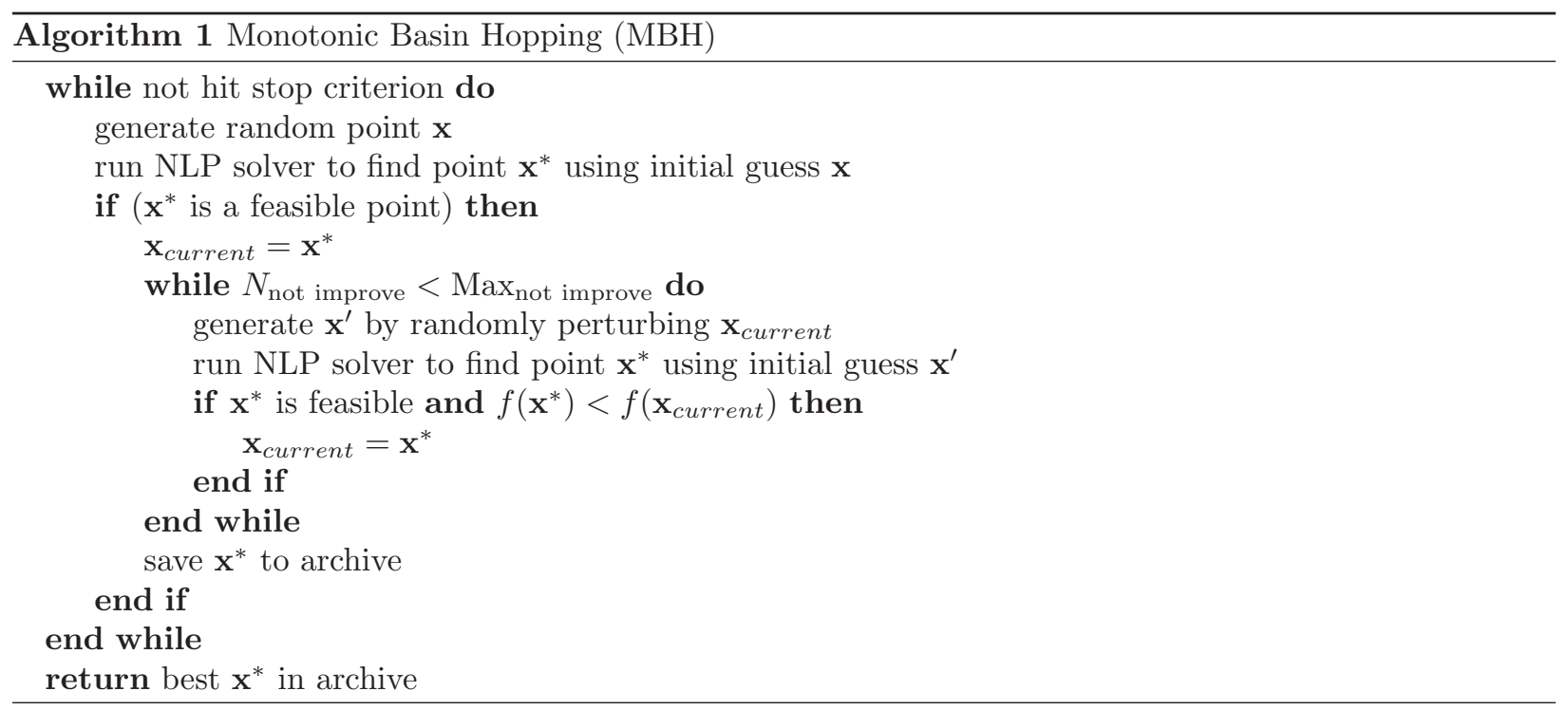

The MBH+SNOPT optimization algorithm in EMTG is efficient and does not require an initial guess. $\mathrm{MBH}$ is most useful when one does not have much a priori information about the solution as is often the case in the MSSR problem. In particular, MBH can find the optimal solution to the variable-mass with spiral descent/ascent modeling MSSR problem when there is no initial guess for the pickup mass (or any other variable in the problem). The combination of the variable mass with spiral descent/ascent modeling formulation with the MBH+SNOPT optimization algorithm is therefore a very valuable tool for MSSR. 


\section{Results}

\section{IV.A. Overview}

Trajectories are designed to each of the three target orbit about Mars using EMTG. Both the FH and SLS departures are evaluated in addition to the 2000 and 3000 s $I_{s p}$ spacecraft configurations. While the the 2000 second configuration increases the spacecraft dry mass and is less efficient than the nominal 3000 second configuration, it provides higher thrust. This additional thrust at the cost of efficiency is often beneficial in short time-of-flight scenarios in which the spacecraft thrusts nearly continuously. In these scenarios the maximum return mass can be limited by the thrust capability of the engine and not the propellant of the spacecraft. For trajectories generated with the higher $I_{s p}$ system, it can be more optimal to depart Earth with less than the maximum allowable propellant.

A range of launch and departure opportunities is inspected, broadening the trade space and allowing for more informed mission planning. The search for optimal return-mass trajectories is segmented into one year launch and one year arrival periods. The one year launch periods for Falcon Heavy departure begin on the September 23rd of 2018, 2019, 2020, 2021, or 2022. The one-year arrival period spans the calendar year from January 1 to December 31, where 2024, 2025, 2026, and 2027 arrival years are investigated. For SLS launches the one-year launch date period begins on July 1. These maximum allowable return mass matrices can then be used to determine when cost and benefit of different launch and arrival date combinations. For certain Earth departure and arrival date periods, gravity assists can improve the maximum allowable return mass. Gravity assists from Earth and Venus are considered for comparison to direct trajectories. Earth gravity assists on ARRM Option B return legs have demonstrated the potential to significantly increase the maximum boulder return mass for some of the NEAs examined, ${ }^{2}$ and are especially beneficial for changing the trajectory plane from the inclination of the asteroid to the ecliptic for Earth-Moon system insertion. For the outbound leg, both Earth and Venus are explored, where gravity assists from those bodies have been shown to improve low-thrust trajectory performance on outbound journeys to Mars. ${ }^{30}$

\section{IV.B. Deimos Results}

Deimos orbits Mars at the highest altitude of the options investigated and, thus, provides the highest feasible return masses. As such, the body provides an upper bound on performance. For trajectory modeling purposes Deimos is assumed to reside in circular orbit about Mars at a radius of $23453 \mathrm{~km}$. Another key spiral modeling assumption is that the spacecraft arrives at Mars sphere of influence with a C3 of zero, and thus the spiral can begin at any Mars inclination. The density of Deimos is $1.48 \mathrm{~g} / \mathrm{cm}^{3},{ }^{31}$ and this value can be used to estimate the size of the boulder size that can be returned from the pickup mass. The stay time at Deimos for proximity operations is constrained to be greater than 200 days, but less than 600 days (also used for Phobos and LMO trajectories).

As noted, different interplanetary options including gravity assists to and from Deimos are explored, namely: EDE (direct), EEDE, EDEE, EVDE, EEDEE, EVDEE, EVEDE, and EVEDEE sequences, where E, D, and V indicate an encounter with Earth (including departure and arrival), Deimos, or Venus respectively. The single Earth gravity assist (outbound or inbound) options are generally more beneficial than trajectories with a Venus gravity assist or a trajectories with a gravity assist on both legs. Trajectory results are listed in Table 2 for a one year launch periods beginning no earlier than (NET) September 23, 2019 and a one year arrival period beginning no later than (NLT) January 1, 2027. For these particular launch and arrival periods, the direct outbound and return leg trajectory outperforms any of the gravity assist scenarios. The return mass from the EEDE sequence is, however, only $200 \mathrm{~kg}$ lower than the direct scenario. The scenarios using a Venus gravity assist do not perform particularly well given the long outbound time-of-flights. High return mass solutions typically benefit from allowing as much flight time as possible for more optimal thrust/coast periods and phasing. Note that the EVDEE and EVEDEE scenarios are either not feasible or return less than $460 \mathrm{~kg}$ of asteroid mass. The trajectories are identified as infeasible in EMTG; however, $460 \mathrm{~kg}$ of hydrazine and xenon is reserved as part of the neutral mass for proximity operations at Deimos. This reserved mass does not need to be returned, and the maximum return mass identified using EMTG can be augmented by $460 \mathrm{~kg}$. Thus, the scenarios may be feasible, but are not competitive in maximum return mass.

Understanding the performance of trajectories over different combinations of departure and arrival dates is of critical importance for the planning of Mars moon or low-Mars orbit sample return mission. The maximum Deimos return mass with different gravity assist scenarios and $I_{s p}$ configurations over the different 
Table 2: Trajectory characteristics and performance for direct and gravity assist scenarios departing Earth NET 9/23/19 (one year period) and returning NLT December 31, 2027 (one year period)

\begin{tabular}{|c|c|c|c|c|c|c|c|c|c|c|}
\hline Scenario & \begin{tabular}{|c|} 
Earth System \\
Departure \\
Date
\end{tabular} & $\begin{array}{c}\text { Deimos } \\
\text { Arrival Date }\end{array}$ & \begin{tabular}{|c|} 
Stay \\
Time \\
(days) \\
\end{tabular} & $\begin{array}{c}\text { Deimos } \\
\text { Departure } \\
\text { Date } \\
\end{array}$ & $\begin{array}{l}\text { Earth Sys tem } \\
\text { Arrival Date }\end{array}$ & $\begin{array}{c}\text { TOF } \\
\text { (years) }\end{array}$ & $\begin{array}{c}\text { Launch } \\
\text { Mass } \\
\text { (kg) }\end{array}$ & \begin{tabular}{|c|} 
Asteroid \\
Arrival \\
Mass (kg) \\
\end{tabular} & \begin{tabular}{|c|} 
Earth \\
Retum \\
Mass (kg) \\
\end{tabular} & $\begin{array}{c}\text { Boulder } \\
\text { Mass } \\
\text { (tons) }\end{array}$ \\
\hline$\overline{\mathrm{EDE}}$ & 7-Jan-2020 & 1-Oct-2022 & 200.0 & $4 / 19 / 2023$ & 31-Oct-2027 & 7.82 & 12242 & 9610 & 15315 & 9.987 \\
\hline EEDE & 3-Jan-2020 & 17-Aug-2022 & 269.4 & 13-May-2023 & $28-0 c t-2027$ & 7.82 & 12024 & 9385 & 15093 & 9.787 \\
\hline EDEE & 29-Sep-2019 & 27-Mar-2022 & 200.0 & 13-Oct-2022 & 8-Feb-2027 & 7.36 & 10919 & 8231 & 13281 & 8.091 \\
\hline EVDE & 20-Nov-2019 & 9-Jun-2024 & 200.0 & 26-Dec-2024 & 8-Oct-2027 & 7.88 & 12810 & 8295 & 11834 & 6.462 \\
\hline EEDEE & 18-Oct-2019 & $28-\mathrm{Feb}-2022$ & 200.0 & 16-Sep-2022 & 29-Jan-2027 & 7.28 & 10190 & 8214 & 13829 & 8.710 \\
\hline EVDEE & \multicolumn{10}{|c|}{ Not feasible or return mass $<460 \mathrm{~kg}$} \\
\hline EVEDE & 23-Sep-2019 & 25-May-2024 & 200.0 & 11-Dec-2024 & 25-Nov-2027 & 8.17 & 11298 & 7897 & 11046 & 5.826 \\
\hline EVEDEE & \multicolumn{10}{|c|}{ Not fea sible or return mass $<460 \mathrm{~kg}$} \\
\hline
\end{tabular}

Earth system departure and arrival periods under consideration are arrayed in Tables 3-5. A "-" in the tables indicates that there are no feasible solutions or the return mass is less than $460 \mathrm{~kg}$ for that date combination. The entries are also color coded according to their relative performance, where the darker the green the higher the maximum return mass. Table 3 outlines the maximum return mass for direct, EDE scenarios for both the 2000 second and 3000 second $I_{s p}$ configuration. The 3000 second $I_{s p}$ configuration generally outperforms the 2000 second $I_{s p}$ configuration except for the 2024 arrival dates, which correspond to short time of flight (TOF) trajectories that benefit from the higher thrust for improved phasing. The EEDE and EDEE trajectories in Tables 4 and 5 follow this same trend, with the 3000 second $I_{s p}$ configuration usually enabling higher return masses except when the TOF is relatively short.

Table 3: Maximum return mass for EDE trajectories with a FH launch and different $I_{s p}$ configurations

Sequence: EDE, Launch vehicle: FH, Isp: 2000s Sequence: EDE, Launch vehicle: FH, Isp: 3000s
Earth system return year
Earth system retum year
(NLT 12/31/YYYY)

Table 4: Maximum return mass for EEDE trajectories with a FH launch and different $I_{s p}$ configurations

\begin{tabular}{|c|c|c|c|c|c|c|c|c|c|c|c|}
\hline \multirow{2}{*}{\multicolumn{6}{|c|}{$\begin{array}{c}\text { Sequence: EEDE, La unch vehicle: FH, Isp: } 2000 \text { s } \\
\text { Earth system return year } \\
\text { (NL T } 12 / 31 / \text { YYYY) }\end{array}$}} & \multicolumn{6}{|c|}{$\begin{array}{c}\text { Sequence: EE DE, Laumch vehicle: FH, Isp: } \mathbf{3 0 0 0 s} \\
\text { Earth system return year }\end{array}$} \\
\hline & & & & & & \multirow{5}{*}{ 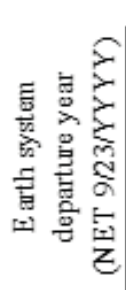 } & & 2024 & 2025 & 2026 & 2027 \\
\hline \multirow{4}{*}{ 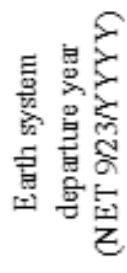 } & 2018 & 1.936 & 8.176 & 7.967 & 7.790 & & 2018 & 0.714 & 8.321 & 6.990 & 9.557 \\
\hline & 2019 & 1.932 & 7206 & 7.122 & 8.566 & & 2019 & 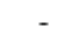 & 8.315 & 6.684 & 9.600 \\
\hline & 202 & & & & & & 2020 & - & - & - & - \\
\hline & 200 & - & - & - & - & & 2021 & - & - & - & 8.479 \\
\hline
\end{tabular}

A mixture of sequences and $I_{s p}$ configurations comprise the best performing cases across the different departure/arrival date combinations. The best Deimos-FH trajectories in terms of return mass for each of the date combinations are compiled in Table 6 and the corresponding trajectory details are listed in Table 7. All trajectories depart with the maximum possible C3 of $2 \mathrm{~km}^{2} / \mathrm{s}^{2}$. Short TOF trajectories (<5.3 years) benefit from the higher thrust of the 2000 second $I_{s p}$ configuration. Note that the launch mass is at its upper bound of $13153 \mathrm{~kg}$ for the 2000 second $I_{s p}$ configuration trajectories to compensate for the higher 
Table 5: Maximum return mass for EDEE trajectories with a FH launch and different $I_{s p}$ configurations

\begin{tabular}{|c|c|c|c|c|c|c|c|c|c|c|c|}
\hline \multicolumn{6}{|c|}{$\begin{array}{c}\text { Sequence: E DEE, L aunch vehicle: FH, Isp: } 2000 \text { s } \\
\text { Earth system return year } \\
\text { (NLT } 12 / 31 / \text { xxx) }\end{array}$} & \multicolumn{6}{|c|}{$\begin{array}{c}\text { Sequence: E DEE, Launch vehicle: FH, Isp: } \mathbf{3 0 0 0 s} \\
\text { Earth system return year } \\
\text { (NLT } 12 / 31 / \mathrm{xxxx} \text { ) }\end{array}$} \\
\hline \multirow{5}{*}{ 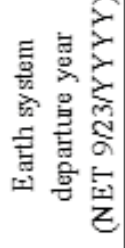 } & & 2024 & 2025 & 2026 & 2027 & \multirow{5}{*}{ 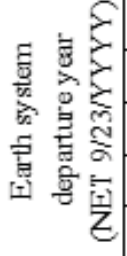 } & & 024 & 5 & 20 & 27 \\
\hline & 2018 & - & .625 & .949 & 7.954 & & 20. & 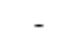 & 4.435 & 9.826 & 9.860 \\
\hline & 2019 & - & - & 7.848 & 7.934 & & 20. & 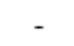 & 4.405 & 8.061 & 8.101 \\
\hline & 2020 & - & - & - & 3.838 & & 20 & - & - & - & 3.260 \\
\hline & 2021 & - & - & - & 4.312 & & 2021 & - & - & - & 2.586 \\
\hline
\end{tabular}

propellant burn rate. On the other hand, optimal trajectories with a 3000 second $I_{s p}$ configuration typically depart with less than the maximum allowable propellant mass to avoid returning to the Earth system with any unused xenon. Notably, an Earth gravity assist on the outbound leg is beneficial for several of these short TOF cases, improving the return mass over the direct scenarios in some departure/arrival date pairs (2018/2024, 2019/2024, 2019/2025, and 2021/2027). With a longer TOF, an Earth gravity assist on the return leg can reduce the propellant required to arrive at the Earth system with a C3 of $2 \mathrm{~km}^{2} / \mathrm{s}^{2}$. An EDEE sequence with a 3000 second $I_{s p}$ is the highest performing scenario for the 2018/2026 and 2019/2026 departure/arrival date combination.

Table 6: Best return mass as a function of departure and arrival period across all Deimos trajectories examined with a $\mathrm{FH}$ launch

\begin{tabular}{|c|c|c|c|c|c|}
\hline \multirow{6}{*}{ 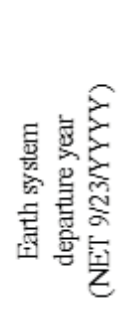 } & & \multicolumn{4}{|c|}{$\begin{array}{c}\text { Earth system retum year } \\
\text { (NLT 12/31/YYYY) }\end{array}$} \\
\hline & & 2024 & 2025 & 2026 & 2027 \\
\hline & 2018 & 1.936 & 9.410 & 9.826 & 10.413 \\
\hline & 2019 & 1.932 & 8.315 & 8.061 & 9.987 \\
\hline & 2020 & - & 0.695 & - & 8.685 \\
\hline & 2021 & - & - & - & 8.479 \\
\hline
\end{tabular}

Table 7: Trajectory characteristics of best return mass cases across all Deimos trajectories examined with a FH launch

\begin{tabular}{|c|c|c|c|c|c|c|c|c|c|c|}
\hline \begin{tabular}{|l|} 
Earth System \\
Departure Date
\end{tabular} & $\begin{array}{l}\text { Earth System } \\
\text { Arrival Date }\end{array}$ & Sequence & $\begin{array}{l}\text { Isp } \\
\text { (sec) }\end{array}$ & $\begin{array}{l}\text { Deimos } \\
\text { Arrival Date }\end{array}$ & \begin{tabular}{|l|} 
Stay \\
time \\
(days)
\end{tabular} & $\begin{array}{l}\text { TOF } \\
\text { (days) }\end{array}$ & $\begin{array}{l}\text { Launch } \\
\text { Mass } \\
\text { (kg) }\end{array}$ & $\begin{array}{l}\text { Deimos } \\
\text { Arrival } \\
\text { M ass } \\
(\mathrm{kg})\end{array}$ & \begin{tabular}{|l} 
Earth \\
Return \\
Mass \\
(kg)
\end{tabular} & \begin{tabular}{|l} 
Max \\
Boulder \\
Mass \\
(tons)
\end{tabular} \\
\hline 20-Aug-2019 & 31-Dec-2024 & EEDE & 2000 & 16-Dec-2021 & 200 & 5.37 & 13153 & 9892 & 7475 & 1.936 \\
\hline 018 & 2025 & EDE & 3000 & 2022 & 200 & 76 & & & 134 & \\
\hline 018 & 2026 & EDEE & 3000 & 2021 & 200 & 7.80 & 12067 & & & 9.826 \\
\hline 019 & 027 & EDE & 3000 & 2022 & 200 & 8.20 & 13096 & 10109 & 827 & 10.413 \\
\hline 019 & 30-De & EEDE & 2000 & 21-Dec-2021 & 200 & 5.26 & 13153 & 73 & 7472 & 1.932 \\
\hline $28-\operatorname{Sep}-2019$ & $20-\operatorname{Sep}-2025$ & EEDE & 3000 & 14-Mar-2022 & 200 & 5.98 & 10244 & 316 & 3439 & 8.315 \\
\hline 28-Sep-2019 & 9-Oct-2026 & EDEE & 3000 & 28-Mar-2022 & 200 & 7.03 & 10967 & 250 & 13255 & 8.061 \\
\hline 7-Jan- 2020 & $31-$ Oct- 2027 & EDE & 3000 & 1-Oct-2022 & 200 & 7.82 & 12242 & 610 & 15315 & 9.987 \\
\hline 4-Dec-2020 & 1-Jun-2025 & EDE & 2000 & 2-Ju1-2023 & 200 & 4.49 & 13153 & 7956 & 6228 & 0.695 \\
\hline 18-Jan-2021 & $25-$ Oct- 2027 & EDE & 3000 & $20-\mathrm{Feb}-2024$ & 200 & 6.76 & 12299 & 8737 & 14014 & 8.685 \\
\hline 28-Dec-2021 & 19-Oct-2027 & EEDE & 3000 & 24-Mar-2024 & 200 & 5.81 & 10906 & 8513 & 13669 & 8.479 \\
\hline
\end{tabular}

The best 2019/2025 trajectory is an EEDE sequence with a 3000 second $I_{s p}$ configuration. That trajectory is plotted in Figure 5, illustrating an energy-raising Earth gravity assist within one year of departure after a period of leverage thrusting. After the flyby, the spacecraft then thrusts continuously (90\% duty cycle) to reach Mars, arriving at the Mars SOI 15.5 months later with a C3 of zero. The spiral down to Deimos orbit requires approximately $1.08 \mathrm{~km} / \mathrm{s}^{2}$ of $\Delta v$ and five months of flight time with starting mass of $8626 \mathrm{~kg}$. The stay time for proximity operations at Deimos is 200 days, during which the spacecraft can retrieve up 
to 8.3 tons from Deimos before continuously thrusting to spiral back out to Mars SOI and back to the Earth system.

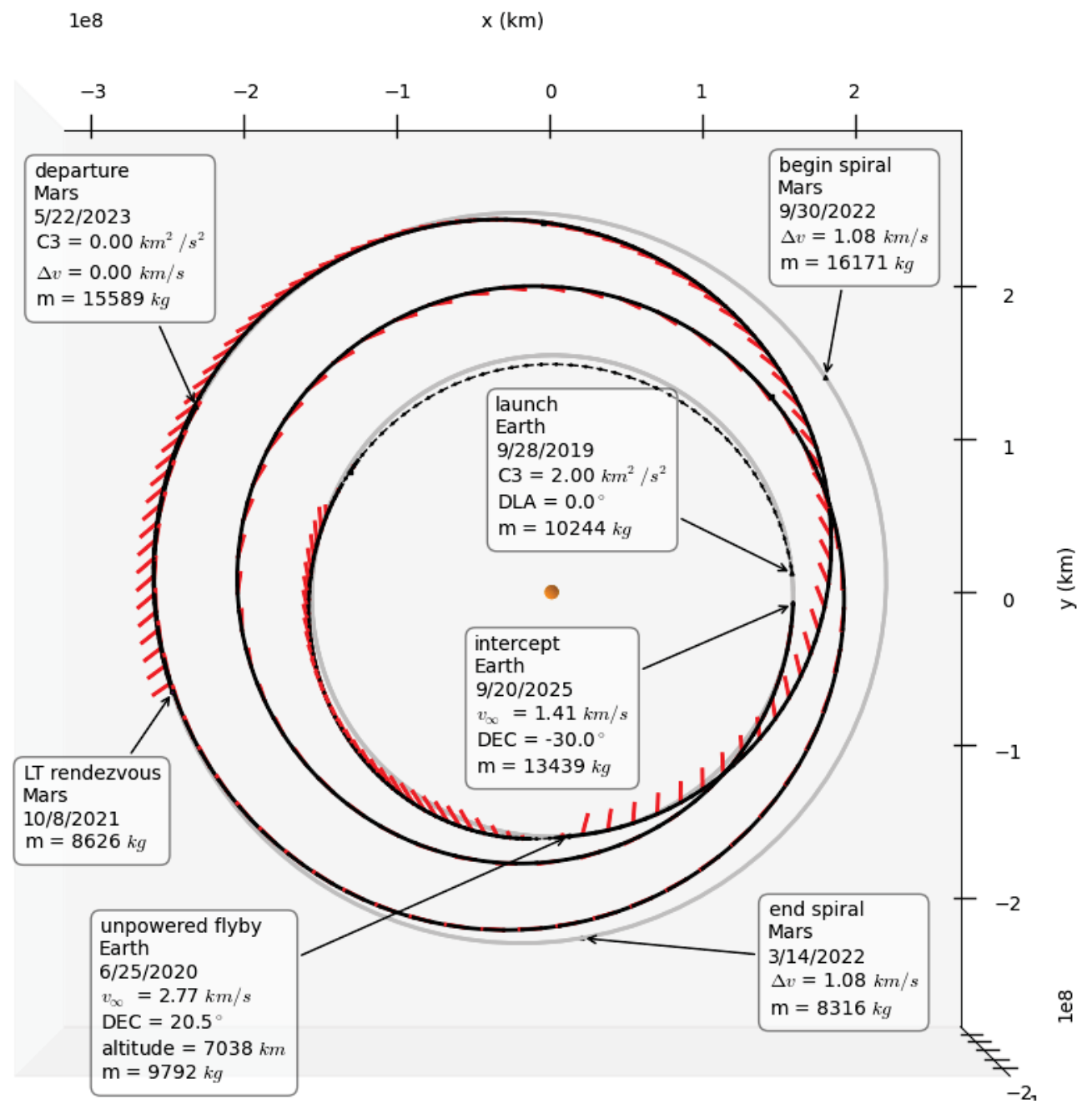

Figure 5: Ecliptic projection of highest Deimos return mass trajectory with a 2019 departure and 2025 arrival for a FH launch (sequence: EEDE, $I_{s p}: 3000$ seconds)

Optimal return-mass trajectories for Deimos are also examined for SLS launches. An Earth gravity assist on the return leg (EDEE) is considered in addition to direct sequences (EDE). Given the high capability of the launch vehicle, a gravity assist on the outbound leg are not examined. Direct and gravity assist return masses from Deimos as a function of one-year Earth launch and arrival date period are outlined in Table 8. The one year launch period begins on July 1 for the SLS cases, and the 2019 launch period cases are not shown as the optimal trajectories are effectively the same trajectory as the 2020 trajectories for corresponding arrival dates. All SLS trajectories are constrained so that the first 30 days of the trajectory is a coasting period, allowing for check-out of the SEP system. Only return masses for the 2000 second $I_{s p}$ configuration are shown as they are higher than the return masses for the 3000 second configuration in all launch and arrival date combinations considered. With the additional launch capability, the SLS is able to deliver more propellant to a higher $\mathrm{C} 3$ and the higher thrust of the 2000 second $I_{s p}$ configuration can be leveraged.

The characteristics of the best performing SLS trajectories are listed in Table 9. In only the longest TOF launch/arrival date pair (2020/2027) is it optimal to launch the maximum spacecraft mass of 16072 $\mathrm{kg}$. This trajectory is the highest performing out of all launch/arrival date pairs with a return mass of over 13 tons, and is plotted in Figure 6. The other scenarios are constrained by the shorter TOF and launching less propellant is optimal. Unlike the FH launch trajectories, gravity assists are not as beneficial for SLS launches, where the EDEE gravity assist trajectory out-performs the direct trajectories in only one date 
combination (2020/2026).

Table 8: Maximum Deimos return mass as a function of departure and arrival period for EDE and EDEE, 2000 second $I_{s p}$ trajectories with a SLS launch

Sequence: EDE, Launch vehicle: SLS, Isp: 2000s Sequence: EDEE, Launch vehicle: SLS, Isp: 2000s
Earth system retum year
Earth system return year
(NLT 12/31/YYYY)
\begin{tabular}{|c|c|c|c|c|c|c|c|c|c|}
\hline & $\mathbf{2 0 2 4}$ & $\mathbf{2 0 2 5}$ & $\mathbf{2 0 2 6}$ & $\mathbf{2 0 2 7}$ \\
\hline $\mathbf{2 0 2 0}$ & 2.097 & 10.661 & 9.049 & 13.454 \\
\hline $\mathbf{2 0 2 1}$ & - & - & - & 11.607 \\
\hline $\mathbf{2 0 2 2}$ & - & - & - & 11.740 \\
\hline
\end{tabular}

Table 9: Trajectory characteristics of best Deimos return mass cases across all departure and arrival date combinations examined with a SLS launch

\begin{tabular}{|c|c|c|c|c|c|c|c|c|c|c|c|}
\hline \begin{tabular}{|c|} 
Earth System \\
Departure \\
Date \\
\end{tabular} & \begin{tabular}{|l} 
E arth System \\
Arrival Date \\
\end{tabular} & Sequence & $\begin{array}{l}\text { Isp } \\
(\mathrm{sec})\end{array}$ & $\begin{array}{c}\mathrm{C} 3 \\
\left(\mathrm{~km}^{\wedge} 2 / \mathrm{s}^{\wedge} 2\right)\end{array}$ & $\begin{array}{c}\text { Deimos } \\
\text { Arrival Date }\end{array}$ & $\begin{array}{c}\text { Stay } \\
\text { time } \\
\text { (days) }\end{array}$ & $\begin{array}{c}\text { TOF } \\
\text { (days) }\end{array}$ & $\begin{array}{c}\text { Launch } \\
\text { Mass } \\
\text { (kg) }\end{array}$ & \begin{tabular}{|c|} 
Deimos \\
Arrival \\
Mass \\
(kg) \\
\end{tabular} & $\begin{array}{c}\text { Earth } \\
\text { Return } \\
\text { Mass } \\
\text { (kg) } \\
\end{array}$ & \begin{tabular}{|c|} 
Max \\
Boulder \\
Mass \\
(tons) \\
\end{tabular} \\
\hline 1-Jul-2020 & 31-Dec-2024 & EDE & 2000 & 8.49 & 17-Dec-2021 & 200 & 4.50 & 12218 & 9892 & 7531 & 2.097 \\
\hline 4-Jul-2020 & 20-Sen-2025 & $\mathrm{EDE}$ & 2000 & 030 & 20-Apr-2022 & 200 & 5.21 & 14217 & 31 & 16323 & 10.661 \\
\hline 1-Jul- & & EDEE & 2000 & 9.00 & & 200 & 6.50 & & & & 11.157 \\
\hline 28-Jul-2020 & 24-Oct-2027 & $\mathrm{EDE}$ & 2000 & 14.58 & 2023 & 200 & 7.24 & 16072 & 10 & 19 & 13.454 \\
\hline 30-Jun-2022 & 6-Oct-2027 & FDF & 2000 & 12.19 & 10-Apr-2024 & 200 & 5.27 & 14917 & 98 & 17 & 11.607 \\
\hline 21-Ju1-2022 & 7-Oct-2027 & $\mathrm{EDE}$ & 2000 & 10.15 & 9-Apr-2024 & 200 & 5.21 & 14833 & 11886 & 17467 & 11.740 \\
\hline
\end{tabular}

\section{IV.C. Phobos Results}

A similar search for high Phobos return mass scenarios across different departure and arrival date combinations is also conducted. The key difference in the design is the additional time and propellant required to spiral down to Phobos orbit radius of $9234 \mathrm{~km}$. The uniform density of Phobos is estimated to be 1.87 $\mathrm{g} / \mathrm{cm}^{3},{ }^{31}$ which is slightly higher than Deimos.

As with Deimos, both direct and gravity assist trajectories are designed for 2000 second and 3000 second $I_{s p}$ configurations. EPE, EEPE, and EPEE scenarios are examined for FH launches, and the return mass from the best performing trajectories for each of the one-year departure/arrival periods is outlined in Table 10. The trajectory characteristics associated with these optimal return masses are listed in Table 11. Notably, the sequences are the same as optimal Deimos trajectories for corresponding departure/arrival date period combinations except for the 2020/2027 pair. The optimal $I_{s p}$ configuration is, however, not the same. Additionally, there are not feasible trajectories that return more than $460 \mathrm{~kg}$ for 2018/2024, 2019/2024, and 2020/2025 pairs because of the additional time and propellant required for spiraling (identified in Table 10 by "-"). Substantial return masses of more than 9 tons are feasible, however, with a departure in 2018 or 2019 and an Earth return in 2027. These two entries are effectively the same trajectory with very similar associated dates and masses. The optimal trajectory associated 2019/2027 entry is plotted if Figure 7. In this trajectory the spacecraft thrusts continuously, with the spiral down to the orbit of Phobos requiring a $\Delta v$ of $1.88 \mathrm{~km} / \mathrm{s}$ and nine months of flight time.

Trajectories to Phobos are also identified for SLS launches. The results across the launch and arrival date periods considered are shown in Table 12. The same constraints and assumptions as for Deimos trajectories are applied. Only direct sequences are explored as it is less likely that a gravity assist on the return leg will offer improvement given the additional time required for spiraling as compared to Deimos. The 2000 second configuration enables higher return masses in all date combinations considered, and the associated trajectory characteristics are outlined in Table 13. As with the best Deimos SLS trajectories, the maximum amount of propellant is launched in the one scenario (2020/2027) associated with the longest TOF. The SLS launch vehicle allows for later departure dates and shorter TOFs as compared to the FH launch trajectories. 


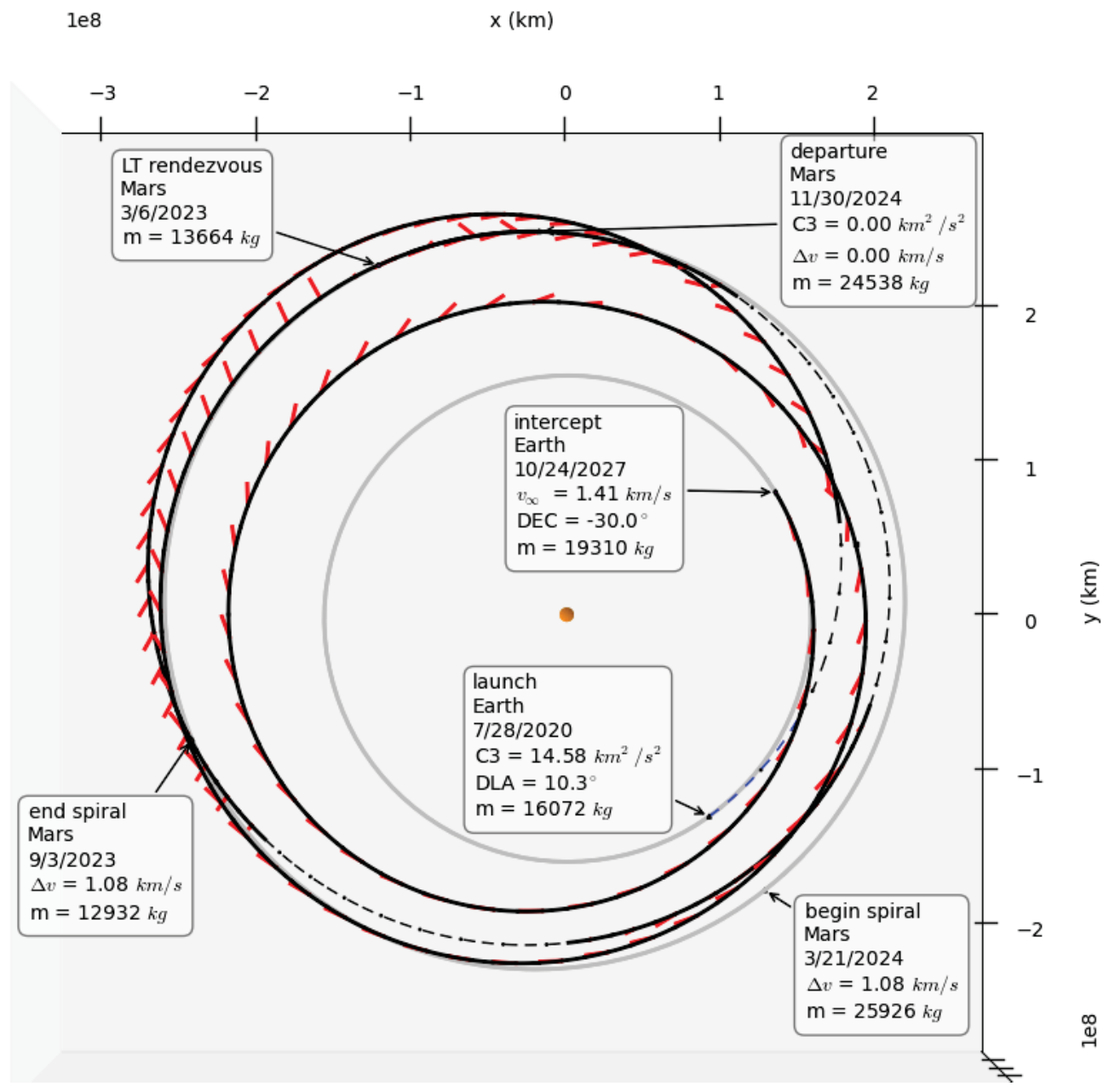

Figure 6: Ecliptic projection of highest Deimos return mass trajectory with a 2020 departure and 2027 arrival for a SLS launch (sequence: EDE, $I_{s p}$ : 2000 seconds)

Table 10: Best return mass as a function of departure and arrival period across all Phobos trajectories examined with a FH launch

\begin{tabular}{c|c|c|c|c|}
\multicolumn{5}{c}{ Earth system return year } \\
(NLT 12/31/YYYY)
\end{tabular}


Table 11: Trajectory characteristics of best return mass cases across all Phobos departure and arrival date combinations examined with a FH launch

\begin{tabular}{|c|c|c|c|c|c|c|c|c|c|c|}
\hline $\begin{array}{c}\text { Earth System } \\
\text { Departure Date }\end{array}$ & \begin{tabular}{|c|} 
Earth System \\
Arrival Date
\end{tabular} & Sequence & \begin{tabular}{|c|} 
Isp \\
(sec)
\end{tabular} & $\begin{array}{c}\text { Phobos } \\
\text { Arrival Date }\end{array}$ & $\begin{array}{c}\text { Stay } \\
\text { time } \\
\text { (days) }\end{array}$ & $\begin{array}{c}\text { TOF } \\
\text { (days) }\end{array} \mid$ & $\begin{array}{c}\text { Launch } \\
\text { Mass } \\
\text { (kg) }\end{array}$ & $\begin{array}{c}\text { Phobos } \\
\text { Arrinal } \\
\text { Mass } \\
\text { (kg) }\end{array}$ & $\begin{array}{c}\text { Earth } \\
\text { Return } \\
\text { M ass } \\
\text { (kg) }\end{array}$ & $\begin{array}{c}\text { Max } \\
\text { Boulder } \\
\text { Mass } \\
\text { (tons) }\end{array}$ \\
\hline 30-Nov-2018 & $15-$ Sep-2025 & EPE & 3000 & 6-Apr-2022 & 200 & 6.80 & 11830 & 8223 & 10519 & 5.249 \\
\hline $25-$ Nov- 2018 & $3-$ Sep-2026 & EPEE & 3000 & 31-Mar-2022 & 200 & 7.77 & 11809 & 8183 & 10599 & 5.331 \\
\hline 22-Sep-2019 & 26-Oct-2027 & EPE & 3000 & 14-Oct-2022 & 200 & 8.09 & 12695 & 9520 & 14508 & 9.137 \\
\hline 1-Oct-2019 & 31-Dec-2025 & EEPE & 3000 & 6-Jun-2022 & 200 & 6.25 & 9972 & 7873 & 8748 & 3.668 \\
\hline 28-Sep-2019 & 17-Nov-2026 & EPEE & 2000 & 23-Aug-2022 & 200 & 7.14 & 13153 & 95 & 10 & 4.551 \\
\hline $27-0$ & $26-\mathrm{Oc}$ & EPE & 3000 & $15-$ Oct- 2022 & 200 & 8.00 & 12546 & & & \\
\hline $15-$ Apr-2021 & & EPE & 2000 & & 208 & 6.38 & 13153 & 10099 & 10964 & 5.407 \\
\hline $23-$ Sep-2021 & 1-Sep-2027 & EEPE & 2000 & 26-Jun-2024 & 231 & 5.94 & 13153 & 9880 & 10394 & 4.840 \\
\hline
\end{tabular}

le8 $\quad x(\mathrm{~km})$

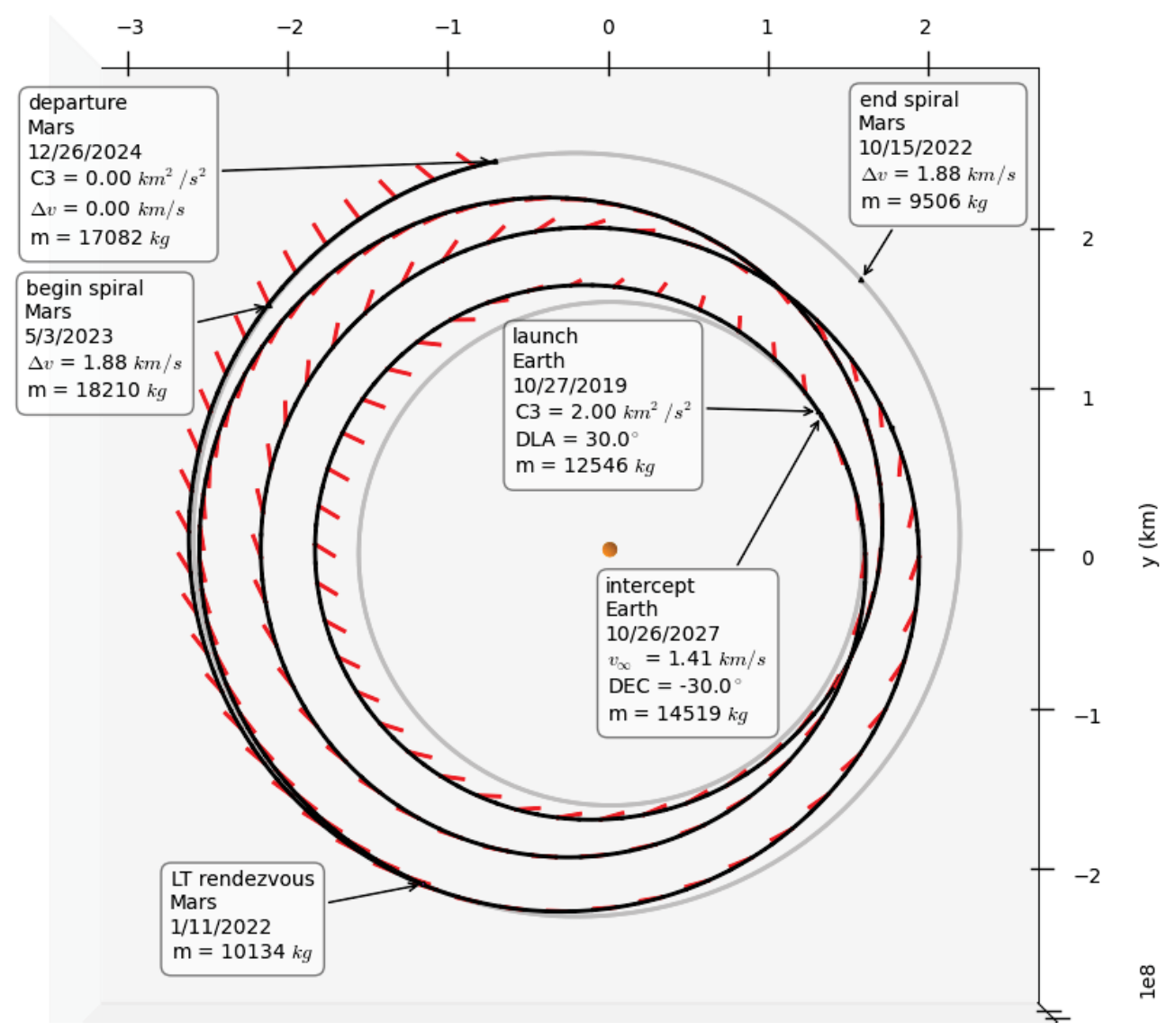

Figure 7: Ecliptic projection of highest Phobos return mass trajectory (2019 departure and 2027 arrival) for a FH launch (sequence: EPE, $I_{s p}: 3000$ seconds) 
Table 12: Maximum Phobos return mass as a function of departure and arrival period for direct 2000 and 3000 second $I_{s p}$ trajectories with a SLS launch

Seque nce: EPE, La unch ve hicle: SLS, Isp: $\mathbf{2 0 0 0 s}$
Earth system return year
(NLT 12/31/YYYY)

Sequence: EPE, La unch rehicle: SLS, Isp: $\mathbf{3 0 0 0 s}$
Earth system return year
(NLT 12/31/YYYY)

Table 13: Characteristics of maximum Phobos return mass trajectories with a 2000 second $I_{s p}$ configuration and a SLS launch

\begin{tabular}{|c|c|c|c|c|c|c|c|c|c|c|c|}
\hline $\begin{array}{c}\text { Earth System } \\
\text { Departure Date }\end{array}$ & \begin{tabular}{|l|} 
Earth System \\
AnrivalDate \\
\end{tabular} & Sequence & $\begin{array}{c}\text { Isp } \\
(\mathrm{sec})\end{array}$ & $\begin{array}{c}\mathrm{C} 3 \\
\left(\mathrm{~km}^{\wedge} 2 / \mathrm{s}^{\wedge} 2\right) \\
\end{array}$ & \begin{tabular}{c|} 
Phobos \\
Arrival Date \\
\end{tabular} & \begin{tabular}{|c|} 
Stay \\
time \\
(days)
\end{tabular} & \begin{tabular}{|l} 
TOF \\
(days)
\end{tabular} & $\begin{array}{c}\text { L aunch } \\
\text { Mass } \\
\text { (kg) }\end{array}$ & \begin{tabular}{|c|} 
Phobos \\
Anrival \\
Mass \\
(kg) \\
\end{tabular} & $\begin{array}{c}\text { Earth } \\
\text { Return } \\
\text { Mass } \\
\text { (kg) }\end{array}$ & $\begin{array}{c}\text { Max } \\
\text { Boulder } \\
\text { Mass } \\
\text { (tons) } \\
\end{array}$ \\
\hline 1-JuL-2020 & 19-Aug-2025 & EPE & 2000 & 8.73 & $20-\sqrt[J u n]{ }-2022$ & 200 & 5.14 & 13575 & 10417 & 11639 & 6.057 \\
\hline 1-Jul-2020 & 1-Jan- 2026 & EPE & 2000 & 8.44 & $25-$ Mhy 2022 & 200 & 5.50 & 13183 & 10142 & 10614 & 5.074 \\
\hline 22-Ju1-2020 & $29-0 \mathrm{ct}-2027$ & EPE & 2000 & 13.87 & 23-Nov- 2023 & 200 & 7.27 & 16072 & 12389 & 16451 & 10.605 \\
\hline $30-$ Jun-2022 & 30-Aug-2027 & EPE & 2000 & 14.03 & $23-J \operatorname{lun}-2024$ & 200 & 5.17 & 14152 & 10731 & 12287 & 6.625 \\
\hline 8-JuL-2022 & 30-Aug-2027 & EPE & 2000 & 13.47 & 23-Jun-2024 & 200 & 5.15 & 14094 & 10729 & 12292 & 6.635 \\
\hline
\end{tabular}

\section{IV.D. LMO Results}

Trajectories are additionally designed to evaluate the ability of the ARRV to spiral down to a $400 \mathrm{~km}$ altitude Mars orbit to retrieve a Mars sample. The same driving assumptions and constraints as in the Deimos and Phobos cases are made, where the $460 \mathrm{~kg}$ of propellant reserved for landing on one the moons is instead used for rendezvous with the sample capsule.

For FH launch scenarios EME, EMEE, and EEME (M indicates Mars for LMO) sequences and 2000 and 3000 second $I_{s p}$ configurations are surveyed. The best return masses from LMO for FH launches as a function of Earth system departure and arrival date period are arrayed in Table 14, and the associated trajectory characteristics are in Table 15. As with Deimos and Phobos, an assortment of different sequences and $I_{s p}$ configurations are represented for FH launches. Gravity assists are again advantageous for improving the maximum return mass, in which five of the eight best trajectories exploit an Earth flyby on the outbound or return leg. Similar trends are also observed on the best $I_{s p}$ configuration for a given departure/arrival period entry, where a 2000 second $I_{s p}$ configuration is better for the shorter TOF cases and a 3000 second $I_{s p}$ configuration is better for the longer TOF scenarios. An early launch and a relatively long TOF enable maximum return masses greater than six tons. If an additional $1700 \mathrm{~kg}$ payload is carried to LMO with the ARRV, 4.8 tons can still be returned from LMO to an Earth-return C3 of $2.0 \mathrm{~km}^{2} / \mathrm{s}^{2}$ for the 2018/2027 and 2019/2027 date combinations. This additional payload could represent a Mars surface descent/retrieval/ascent system, enabling a Mars sample return with a single FH launch.

Table 14: Best return mass as a function of departure and arrival period across all LMO trajectories examined with a FH launch

\begin{tabular}{c|c|c|c|c|}
\multicolumn{9}{c}{ Earth system return year } \\
\hline & $\mathbf{2 0 2 4}$ & $\mathbf{2 0 2 5}$ & $\mathbf{2 0 2 6}$ & $\mathbf{2 0 2 7}$ \\
\hline $\mathbf{2 0 1 8}$ & - & 1.280 & 1.323 & 6.225 \\
\hline $\mathbf{2 0 1 9}$ & - & 1.101 & 0.918 & 6.224 \\
\hline $\mathbf{2 0 2 0}$ & - & - & - & 0.588 \\
\hline $\mathbf{2 0 2 1}$ & - & - & - & 0.889 \\
\hline
\end{tabular}

18 of 22

American Institute of Aeronautics and Astronautics 
Table 15: Trajectory characteristics of best return mass cases across all LMO departure and arrival date combinations examined with a FH launch

\begin{tabular}{|c|c|c|c|c|c|c|c|c|c|c|}
\hline $\begin{array}{c}\text { Earth System } \\
\text { Departure Date }\end{array}$ & $\begin{array}{c}\text { Earth System } \\
\text { Arrival Date }\end{array}$ & Sequence & $\begin{array}{c}\text { Isp } \\
\text { (sec) }\end{array}$ & $\begin{array}{c}\text { LMO Arrival al } \\
\text { Date }\end{array}$ & $\begin{array}{c}\text { Stay } \\
\text { time } \\
\text { (days) }\end{array}$ & $\begin{array}{c}\text { TOF } \\
\text { (days) }\end{array}$ & $\begin{array}{c}\text { Launch } \\
\text { M ass } \\
\text { (kg) }\end{array}$ & $\begin{array}{c}\text { LMO } \\
\text { Arrival } \\
\text { M ass } \\
\text { (kg) }\end{array}$ & $\begin{array}{c}\text { Earth } \\
\text { Return } \\
\text { M ass } \\
\text { (kg) }\end{array}$ & $\begin{array}{c}\text { M ax } \\
\text { Boulder } \\
\text { Mass } \\
\text { (tons) }\end{array}$ \\
\hline \hline 16-May-2019 & 22-Jun-2025 & EEME & 2000 & 8-Sep-2022 & 200 & 6.10 & 13153 & 9116 & 6816 & 1.280 \\
\hline 14-Nov-2018 & $25-$-Oct-2026 & EMEE & 3000 & 5-Ju-2022 & 200 & 7.95 & 11260 & 7405 & 6523 & 1.323 \\
\hline 23-Sep-2019 & 6-Oct-2027 & EEME & 3000 & 2-Ju-2023 & 200 & 8.04 & 12277 & 9192 & 11543 & 6.225 \\
\hline 23-Sep-2019 & 23-Jun-2025 & EEME & 2000 & 17-Sep-2022 & 200 & 5.75 & 13153 & 9022 & 6636 & 1.101 \\
\hline 27-Sep-2019 & 8-Oct-2026 & EMEE & 2000 & 16-Oct-2022 & 200 & 7.03 & 13153 & 8639 & 6452 & 0.918 \\
\hline 18-Jan-2020 & 6-Oct-2027 & EME & 3000 & 4-Ju-2023 & 200 & 7.72 & 12684 & 9244 & 11583 & 6.224 \\
\hline 23-Sep-2021 & 7-Jul-2027 & EME & 2000 & 14-Nov-2024 & 200 & 5.79 & 13153 & 8809 & 6120 & 0.588 \\
\hline 3-Ju-2022 & 8-Jul-2027 & EME & 2000 & 22-Oct-2024 & 200 & 5.01 & 13153 & 8938 & 6423 & 0.889 \\
\hline
\end{tabular}

Returns from LMO are viable for TOFs as short as five years with a FH launch. The best 2021/2027 trajectory has a 5.01 year TOF and is plotted in Figure 8. Interestingly, the outbound and return leg legs are nearly symmetric in shape with Mars line of apsides. This geometry enables the spiraling period down to LMO and back to Mars SOI to occur around Mars perihelion instead of aphelion, allowing for higher power to the SEP system. The spiral down to LMO requires a $\Delta v$ of $3.09 \mathrm{~km} / \mathrm{s}$ and $10.5 \mathrm{months}$. After the sample is retrieved the spiral back up to SOI takes 11.5 months. Recall that the arrays degrade throughout the trajectory decreasing the power available.

SLS launch scenarios to LMO are designed as well. As with Phobos, only direct sequences are examined for both $I_{s p}$ configurations. The maximum return mass as a function of launch and Earth system arrival date period are illustrated in Table 16. The trajectory characteristics of the best performing cases for the launch/arrival periods are listed in Table 17. Unlike the Phobos SLS cases there is a 3000 second $I_{s p}$ configuration scenario that enables a higher return mass than the 2000 second $I_{s p}$ configuration. Returns are feasible for the same launch/arrival date periods as Phobos SLS cases, but are significantly lower. Despite the long spiral times, over six tons can be returned from LMO for a seven year TOF.

Table 16: Maximum LMO return mass as a function of departure and arrival period for EME, 2000 and 3000 second $I_{s p}$ trajectories with a SLS launch

Sequence: EME, Launch v ehicle: SLS, Isp: 2000s
Earth system return year
(NLT 12/31/YYYY)

Sequence: EME, La unch vehicle: SLS, Isp: 3000s
Earth system return year
(NL T 12/31/YYYY)

Table 17: Trajectory characteristics of best LMOreturn mass cases across all departure and arrival date combinations examined with a SLS launch

\begin{tabular}{|c|c|c|c|c|c|c|c|c|c|c|c|}
\hline $\begin{array}{c}\text { Earth Sys tem } \\
\text { Departure Date }\end{array}$ & $\begin{array}{l}\text { Earth System } \\
\text { Arrival Date }\end{array}$ & Sequence & $\begin{array}{c}\text { Isp } \\
\text { (sec) }\end{array}$ & $\begin{array}{c}\mathrm{C} 3 \\
\left(\mathrm{~km}^{\wedge} 2 / \mathrm{s}^{\wedge} 2\right)\end{array}$ & $\begin{array}{c}\text { LMO Arrival } \\
\text { Date }\end{array}$ & $\begin{array}{c}\text { Stay } \\
\text { time } \\
\text { (days) }\end{array}$ & $\begin{array}{c}\text { TOF } \\
\text { (days) }\end{array}$ & $\begin{array}{c}\text { Launch } \\
\text { Mass } \\
\text { (kg) }\end{array}$ & $\begin{array}{c}\text { LMO } \\
\text { Arrival } \\
\text { Mass } \\
\text { (kg) }\end{array}$ & $\begin{array}{c}\text { Earth } \\
\text { Return } \\
\text { Mass } \\
\text { (kg) }\end{array}$ & $\begin{array}{c}\text { Max } \\
\text { Bouller } \\
\text { Mass } \\
\text { (tons) }\end{array}$ \\
\hline 1-Jul-2020 & 7-Jun-2025 & EME & 2000 & 8.47 & 8-Sep-2022 & 200 & 4.93 & 12553 & 9100 & 6903 & 1.426 \\
\hline 1-Jul-2020 & 1-Jun-2026 & EME & 2000 & 8.48 & 23-Aug-2022 & 200 & 5.92 & 12323 & 8934 & 6211 & 0.760 \\
\hline 21-Aug -2020 & 7-Oct- 2027 & EME & 3000 & 12.22 & 3-Ju1-2023 & 200 & 7.12 & 11548 & 9151 & 11653 & 6.406 \\
\hline 19-Jun-2022 & 9-Ju1-2027 & EME & 2000 & 20.28 & $26-$ Sep-2024 & 200 & 5.06 & 12901 & 9130 & 6823 & 1.313 \\
\hline 1-JuL-2022 & 10-Ju1-2027 & EME & 2000 & 19.37 & $26-$ Sep- 2024 & 200 & 5.03 & 12855 & 9126 & 6820 & 1.313 \\
\hline
\end{tabular}




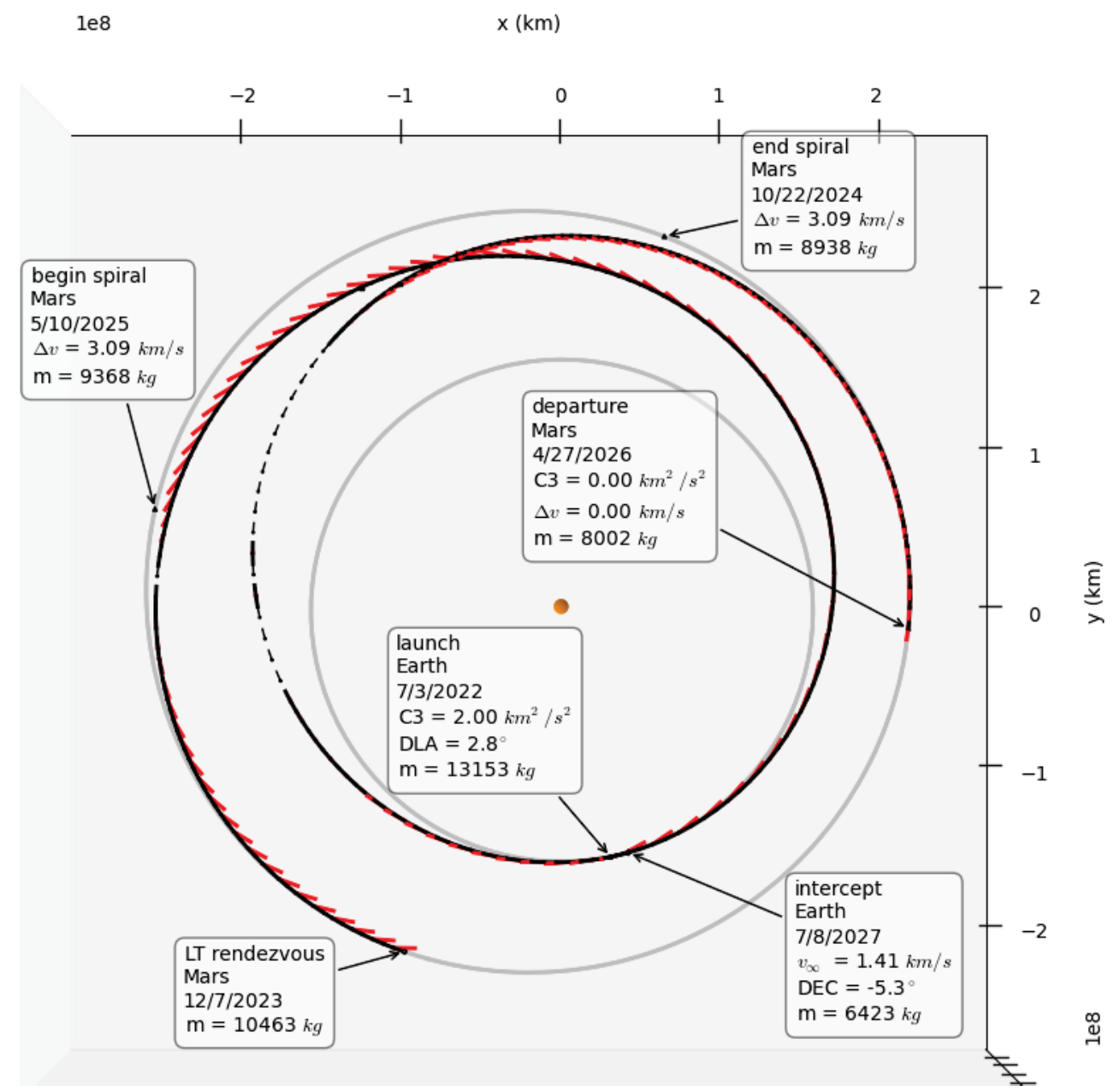

Figure 8: Ecliptic projection of shortest time-of-flight trajectory from best LMO return mass trajectories (2021 departure and 2027 arrival) for a FH launch (sequence: EME, $I_{s p}$ : 2000 seconds) 


\section{Conclusion}

\section{V.A. Summary}

In this work, a design technique was developed for the MSSR mission which allows the mission design tool to model operations at Mars and directly optimize the return mass without needing a large number of parametric runs. This technique is highly efficient and significantly reduces the human work-load necessary to design such missions. The algorithms presented in this work have been implemented in Goddard's low-thrust trajectory design tool EMTG, which is available open-source from http://sourceforge net/projects/emtg/.

The technique developed here was then used to design several different variants of MSSR, including missions to Deimos, Phobos, and LMO using two different SEP systems and two different launch vehicles - FH and SLS. Many viable MSSR trajectories were found with launch dates between 2018 and 2021 and return to cislunar space between 2025 and 2027. These results show that it is possible to return as much as 13 tons of material from Deimos, 11 tons from Phobos, and 6 tons from LMO. In several scenarios an Earth gravity assist on the outbound or return leg can increase the return mass from trajectories following a direct sequence for the same launch and arrival period. The ARRV is shown to be a highly flexible vehicle, capable of accomplishing the MSSR mission as well as the ARRM Option B mission for which it was originally designed.

\section{V.B. Future Work}

As the results of this study show, there is a broad space of possible trajectory options for MSSR and also many possible combinations of the system parameters such as propulsion and launch vehicle options. The problem space might be further expanded by investigating several different solar array sizes. The full-problem trajectory optimization technique introduced in this work significantly reduces the work load required to evaluate a given problem but there are opportunities for further automation that will allow the problem space to be more thoroughly explored in a short period of time.

The full MSSR problem involves trading over launch year, arrival year, launch vehicle, propulsion system, solar array size, possible planetary flybys, and return mass to produce a non-dominated hyper-surface among these parameters. Future work by these authors will investigate fully autonomous methods of finding that surface.

\section{References}

\footnotetext{
${ }^{1}$ Brophy, J. R., R., G., Landau, D., Yeomans, D., Polk, J., Porter, C., Williams, W., Allen, C., and Asphaug, E.

${ }^{2}$ Merrill, R. and et al., "Interplanetary Trajectory Design for the Asteroid Robotic Redirect Mission Alternative Approach Trade Study," AIAA/AAS Astrodynamics Specialist Conference, August 2014.

${ }^{3}$ Sweetser, T., Phobos First!-The right focus for NASA's vision, 2012, p. 4241.

${ }^{4}$ Landis, G., "Teleoperation from Mars orbit: A proposal for human exploration," Acta Astronautica, Vol. 62, No. 1, January 2008, pp. 59-65.

5 "Vision and Voyages for Planetary Science in the Decade 20132022," Tech. rep., Space Studies Board, 2011.

${ }^{6}$ Englander, J., Conway, B., and Williams, T., "Automated Mission Planning via Evolutionary Algorithms," Journal of Guidance, Control, and Dynamics, Vol. 35, No. 6, 2012, pp. 1878-1887.

${ }^{7}$ Englander, J. A., Conway, B. A., and Williams, T., "Automated Interplanetary Mission Planning," AAS/AIAA Astrodynamics Specialist Conference, Minneapolis, MN, August 2012.

${ }^{8}$ Englander, J. A., Automated Trajectory Planning for Multiple-Flyby Interplanetary Missions, Ph.D. thesis, University of Illinois at Urbana-Champaign, April 2013.

${ }^{9}$ Ellison, D. H., Englander, J. A., and Conway, B. A., "Robust Global Optimzation of Low-Thrust, Multiple-Flyby Trajectories," AAS/AIAA Astrodynamics Specialist Conference, Hilton Head, SC, August 2013.

${ }^{10}$ Englander, J. A., Ellison, D. H., and Conway, B. A., "Global Optimization of Low-Thrust, Multiple-Flyby Trajectories at Medium and Medium-High Fidelity," AAS/AIAA Space-Flight Mechanics Meeting, Santa Fe, NM, January 2014.

${ }^{11}$ Ellison, D. H., Englander, J. A., Ozimek, M. T., and Conway, B. A., "Analytical Partial Derivative Calculation of the Sims-Flanagan Transcription Match Point Constraints," AAS/AIAA Space-Flight Mechanics Meeting, Santa Fe, NM, January 2014.

${ }^{12}$ Englander, J. A. and Englander, A. C., "Tuning Monotonic Basin Hopping: Improving the Efficiency of Stochastic Search as Applied to Low-Thrust Trajectory Optimization," 24th International Symposium on Space Flight Dynamics, Laurel, MD, May 2014.

${ }^{13}$ McElrath, T. P., Lantoine, G., Landau, D., Grebow, D., Strange, N., Wilson, R., , and Sims, J., "Using Gravity-Assists in the Earth-Moon System as a Gateway to the Solar System," Global Exploration Conference, May 2012.

${ }^{14}$ Ocampo, C., Elements of a Software System for Spacecraft Trajectory Optimization, Cambridge University Press, 2010, pp. $79-111$.
} 
${ }^{15}$ Sims, J. A. and Flanagan, S. N., "Preliminary Design of Low-Thrust Interplanetary Missions," AAS/AIAA Astrodynamics Specialist Conference, Girdwood, Alaska, August 1999.

${ }^{16}$ Sims, J., Finlayson, P., Rinderle, E., Vavrina, M., and Kowalkowski, T., "Implementation of a low-thrust trajectory optimization algorithm for preliminary design," AIAA/AAS Astrodynamics Specialist Conference, August 2006. 2005.

${ }^{17}$ McConaghy, T. T., GALLOP Version 4.5 User's Guide, School of Aeronautics and Astronautics, Purdue University,

18 "PaGMO (Parallel Global Multiobjective Optimizer)," March 2012, http://pagmo.sourceforge.net/pagmo/index.html.

${ }^{19}$ Prussing, J. and Conway, B., Orbital Mechanics, Oxford University Press, New York, 1993.

${ }^{20}$ Conway, B. A., "An Improved Method due to Laguerre for the Solution of Kepler's Equation," Celestial Mechanics, Vol. 39, No. 2, 1986, pp. 199-211.

${ }^{21}$ Edelbaum, T., "Propulsion Requirements for Controllable Satellites," ARS Journal, Vol. 31, No. 8, 1961, pp. $1079-1089$.

${ }^{22}$ Raowulf, G. and Coverstone, V., "Near-Optimal Low-Thrust Orbit Transfers Generated by a Genetic Algorithm," Journal of Spacecraft and Rockets, Vol. 33, No. 6, 1996, pp. 859-862.

${ }^{23}$ Coverstone-Carroll, V., "Near-Optimal Low-Thrust Trajectories via Micro-Genetic Algorithms," Journal of Guidance Control and Dynamics, Vol. 20, 1997, pp. 196-198.

${ }^{24}$ Coverstone-Carroll, V., Hartmann, J., and Mason, W., "Optimal multi-objective low-thrust spacecraft trajectories," Computer Methods in Applied Mechanics and Engineering, Vol. 186, No. 24, 2000, pp. 387 - 402.

${ }^{25}$ Vavrina, M. and Howell, K., "Global Low Thrust Trajectory Optimization through Hybridization of a Genetic Algorithm and a Direct Method," AIAA/AAS Astrodynamics Specialist Conference and Exhibit, Honolulu, Hawaii, August 18-21 2008.

${ }^{26}$ Yam, C., di Lorenzo, D., and Izzo, D., "Low-Thrust Trajectory Design as a Constrained Global Optimization Problem," Proceedings of the Institution of Mechanical Engineers, Part G: Journal of Aerospace Engineering, Vol. 225, 2011, pp. 12431251.

${ }^{27}$ Leary, R., "Global optimization on funneling landscapes," Journal of Global Optimization, Vol. 18, No. 4, December 2000, pp. 367-383.

${ }^{28}$ Vasile, M., Minisci, E., and Locatelli, M., "Analysis of Some Global Optimization Algorithms for Space Trajectory Design," Journal of Spacecraft and Rockets, Vol. 47, No. 2, March-April 2010, pp. 334-344.

${ }^{29}$ Addis, B., Cassioli, A., Locatelli, M., and Schoen, F., "A global optimization method for the design of space trajectories," Computational Optimization and Applications, Vol. 48, No. 3, April 2011, pp. 635-652.

${ }^{30}$ Williams, S. and Longuski, J., "Low Energy Trajectories to Mars via Gravity Assist from Venus and Earth," Journal of Spacecraft and Rockets, Vol. 28, No. 4, 1991, pp. 486-488.

${ }^{31}$ Rosenblatt, P., "The origin of the Martian moons revisited," The Astronomy and Astrophysics Review, Vol. 19, 2011, pp. 44. 